Review

\title{
The utilization of leaf-based adsorbents for dyes removal: A review
}

\author{
Laura Bulgariu $^{\mathrm{a}, *}$, Leticia Belén Escudero ${ }^{\mathrm{b}, *}$, Olugbenga Solomon Bello ${ }^{\mathrm{c}, *}$, Munawar Iqbal ${ }^{\mathrm{d}, *}, \operatorname{Jan}_{\text {Nisar }}^{\mathrm{e}}$, \\ Kayode Adesina Adegoke c,f, Fadi Alakhras ${ }^{\text {g,* }}$, Michael Kornaros ${ }^{\text {h,i,*, }}$, Ioannis Anastopoulos ${ }^{\text {h,* }}$
}

${ }^{a}$ Technical University Gheorghe Asachi of Iasi, Faculty of Chemical Engineering and Environmental Protection, Department of Environmental Engineering and Management, D. Mangeron, 71A, 700050 Iasi, Romania

${ }^{\mathrm{b}}$ Laboratory of Analytical Chemistry for Research and Development (QUIANID), Interdisciplinary Institute of Basic Sciences (ICB), UNCUYO-CONICET, Faculty of Natural and Exact Sciences, National University of Cuyo, Padre J. Contreras 1300, 5500 Mendoza, Argentina

${ }^{c}$ Department of Pure and Applied Chemistry, Ladoke Akintola University of Technology, P. M. B. 4000, Ogbomoso, Oyo State, Nigeria

d Department of Chemistry, University Lahore, Lahore, Pakistan

${ }^{\mathrm{e}}$ National Centre of Excellence in Physical Chemistry, University of Peshawar, Peshawar 25120, Pakistan

${ }^{\mathrm{f}}$ Department of Chemistry, University of Pretoria, Pretoria 0002, South Africa

${ }^{\mathrm{g}}$ Department of Chemistry, College of Science, Imam Abdulrahman Bin Faisal University, P.O. Box 1982, Dammam 31441, Saudi Arabia

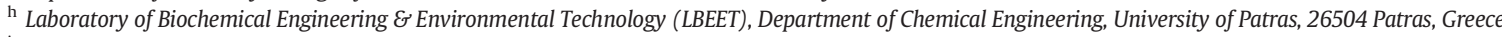

i INVALOR: Research Infrastructure for Waste Valorization and Sustainable Management, 1 Karatheodori Str., University Campus, 26504, Patras, Greece

\section{A R T I C L E I N F O}

\section{Article history:}

Received 28 August 2018

Received in revised form 19 November 2018

Accepted 1 December 2018

Available online 7 December 2018

\section{Keywords:}

Leaves

Characterization

Modification

Equilibrium modeling

Thermodynamic studies

Dyes

\begin{abstract}
A B S T R A C T
The presence of organic dyes in the aquatic environment is a serious global problem because of the serious negative consequences on the quality of ecosystems. Among various physico-chemical methods, the adsorption could be considered a promising alternative for removing dyes from aqueous media, due to its efficiency, high selectivity, low cost, ease of operation, simplicity, and availability in a wide range of experimental conditions. However, all these advantages are closely related to the nature of adsorbent material used in the adsorption processes of dyes. The adsorbent materials available in large quantities requiring a simple preparation will enhance the benefits of the adsorption processes, in agreement with the concepts of green chemistry. This review is focused on the use of leaf-based materials, in raw or modified forms, as adsorbents for the removal of dyes from aqueous effluents, with applications in the wastewater treatment. This review addresses characterization of leaf-based adsorbents, possible utilization of leaf-based adsorbents (raw and activated forms) for dye removal and possible applications in pilot and full scale systems. Also, thermodynamics, equilibrium and kinetic parameters of dye adsorption on leaf-based adsorbents are discussed. The practical utility of leaf-based adsorbents for dye removal, and their possible uses in the treatment of industrial wastewater are copiously highlighted.
\end{abstract}

(c) 2018 Elsevier B.V. All rights reserved.

\section{Contents}

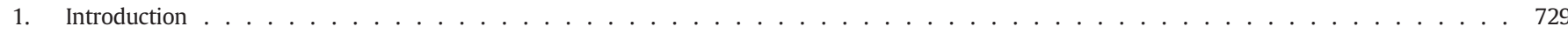

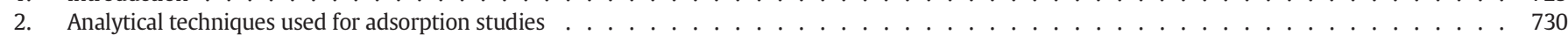

2.1. Scanning electron microscopy . . . . . . . . . . . . . . . . . . . . . . . . . . . . . . . 730

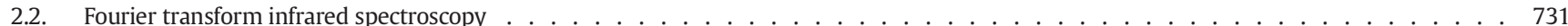

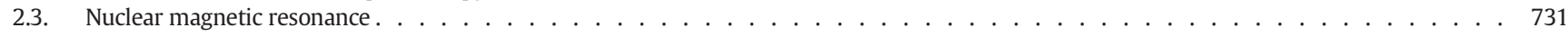

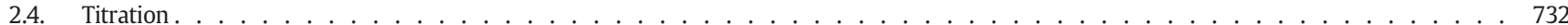

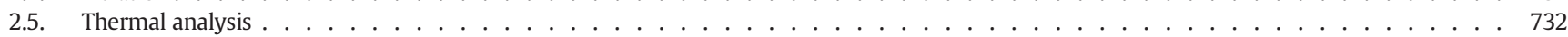

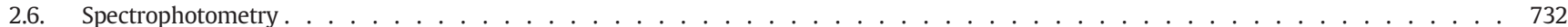

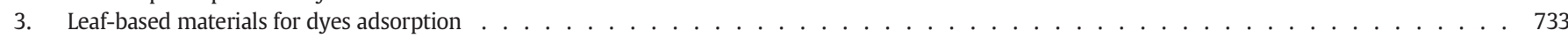

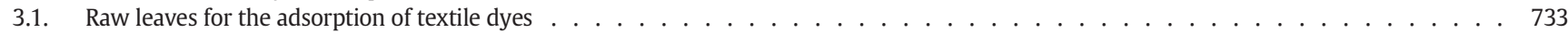

3.2. Carbonaceous adsorbents (such as carbonized materials, activated carbons etc.) derived from leaves . . . . . . . . . . . . . . . . . . 737

3.3. Modified leaf-derived adsorbents . . . . . . . . . . . . . . . . . . . . . . . . . . . 739

4. Thermodynamic studies . . . . . . . . . . . . . . . . . . . . . . . . . . . . . . . . . .

5. Conclusions, future perspectives and challenges . . . . . . . . . . . . . . . . . . . . . . . . . . . . . . . . . 744

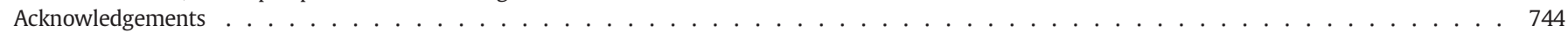

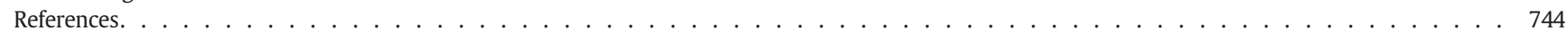

\footnotetext{
* Corresponding authors.

E-mail addresses: lbulg@ch.tuiasi.ro (L. Bulgariu), letibelescudero@gmail.com (L.B. Escudero), osbello06@gmail.com (O.S. Bello), munawar.iqbal@chem.uol.edu.pk (M. Iqbal), falakhras@iau.edu.sa (F. Alakhras), kornaros@chemeng.upatras.gr (M. Kornaros), anastopoulos_ioannis@windowslive.com (I. Anastopoulos).
} 


\section{Nomenclature}

\section{Adsorbents}

Aegle marmelos leaves AMP

Artocarpus heterophyllus leaf powder AHLP

Ashoka plant leaf powder APLP

Pineapple leaf powder PLP

Platanus orientalis leaves powder POLP

Princess tree leaf PTL

Salix babylonica leaves powder SBLP

Water bamboo leaves WBL

Moringa oleifera leaf $\mathrm{MOL}$

$\mathrm{FeCl}_{3}$-activated carbon

FAC

Guava leaf activated carbon GLAC

Activated carbons obtained berry leaves impregnated with $\mathrm{H}_{3} \mathrm{PO}_{4}$ HMBL

Durian leaf powder modified with $\mathrm{NaOH}$ NaOH-DLP

Citric acid-modified Ricinus communis leaves

CALRC

Surfactant-modified-pineapple leaf powder

SMPLP

HCl-modified Calotropis procera leaf powder

$\mathrm{HCl}-\mathrm{ACLP}$

$\mathrm{NaOH}$-modified C. camphora leaves powder

$\mathrm{N}-\mathrm{CLP}$

Modified-Glossogyne tenuifolia leaves

MGTL

Imperata cylindrica activated carbon ICAC

Adsorption related terms

Artificial Neural Network ANN

Bed depth service time BDST

Freundlich $\mathrm{F}$

Langmuir $\mathrm{L}$

Pseudo-first order

PFO

Pseudo-second order

PSO

Intraparticle diffusion (IPD)

Thomas Th

Yoon-Nelson

Y-N

\section{Analytical techniques}

Differential scanning calorimetry DSC

Fourier transform infrared spectroscopy FTIR

Nuclear magnetic resonance NMR

Scanning electron microscopy SEM

Thermogravimetric analysis TGA

Transmission Electron Microscopy TEM

Dyes

Acid Blue 25 AB25

Acid Blue 113 AB113

Acid Green 25 AG25

Acid Orange 52 A052

Acid red 27 AR27

Acid violet 17 AV17

Basic Red 46 BR46

Brill Red 5B BR5B

Brilliant green BG

Congo red CR

Crystal violet CV

Eriochrome Black T EBT

Malachite green MG

Methylene blue MB
Methyl orange MO

Methyl violet MV

Orange G OG

Rhodamine B RhB

Others

The $\mathrm{pH}$ value of the point of zero charge $\mathrm{pHpzc}$

Arrhenius activation energy of adsorption $E_{\mathrm{a}}$

Hexadecyltrimethylammonium bromide HDTMA-Br

\section{Introduction}

One of the most important challenges that occur worldwide is the rapid increase in water consumption due to population growth, urbanization and industrialization. Daily, the water resources are contaminated by industrial effluents, and this affect both the quality of ecosystems and the health of all living forms [1]. One of the most relevant categories of environmental pollutants is the one caused by dyes, which are present in large amount in the wastewater from various industrial activities such as textile, dying, tanner, pulp and paper, paint and pigments [2]. Nowadays, there are $>100,000$ commercial dyes (anionic, cationic or non-ionic) with a rough estimated production of $10^{6}$ tons per year [3]. However, recent studies reported that over $15 \%$ from total quantity of dyes produced annually are lost during the production processes and reach industrial wastewater, becoming contaminants of the environment $[4,5]$.

Beside their well known toxic and carcinogenic effects [6,7], the presence of dyes in aquatic environment causes the water colouring and limits the oxygenation of water surfaces, significantly affecting aquatic plants and fauna $[8,9]$. In addition, because most of dyes used in industrial activities are synthetic dyes (due to their low cost and high chemical stability) $[10,11]$, which contains in the chemical structure aromatic rings, their degradation into environment required long periods of time. Also, the synthetic dyes can generate during of the degradation, secondary toxic products, which are harmful to the environment [12]. Therefore, the effective removal of these hazardous dyes from industrial wastewater before being discharge into environment is a serious problem whose resolution, besides helping to improve the quality of aquatic ecosystems, can also bring important economic benefits.

Various physico-chemical or biological methods can be used for to remove dyes from aqueous media, including chemical precipitation [13], ultrafiltration [14], aerobic and anaerobic microbial degradation [15], coagulation/flocculation [16], advanced oxidation processes [17], electrochemical treatments [18], reverse osmosis [19], and adsorption [6]. In the last period, it has also been shown that some advanced alternatives such as photocatalytic oxidation [20-22] and adsorption processes that use magnetic nano-particles [23], have a high efficiency in removing dyes from aqueous effluents. Although most of these methods have proven their effectiveness in the removing of dyes at laboratory level, they have limited industrial applications mainly because are expensive, required high energy consumption and operation time, and in some cases can generate large quantities of secondary sludge, which must be also properly treated in order to prevent the environment contamination [3]. Therefore, in the selection of appropriate method in terms of environment and economy, not only the efficiency in the removal of dyes from wastewater is important, but also the required raw materials and costs have a decisive role.

Unlike the other methods, adsorption could be considered a promising alternative for removing dyes from aqueous media due to its efficiency, high selectivity, low cost, ease of operation, simplicity, availability in a wide range of experimental conditions, among other advantages $[3,24,25]$. But, all these advantages are closely related to the nature of adsorbent material used in the dyes adsorption processes. Thus, a readily available adsorbent material requiring a simple 
preparation process will enhance the benefits of the adsorption process and will recommend this method for a possible widespread use.

This is the reason for that in recent years, many adsorbent materials obtained from natural materials (such as clay minerals) [26], agricultural waste and by-products (e.g. coffee waste, sugarcane bagasse, agricultural peels) $[5,27,28]$, or industrial waste and by-products (such as aluminium oxide, fly ash, plastics, etc.) $[29,30]$ have been proposed in literature for the adsorptive removal of ionic and non-ionic dye molecules from aqueous solution. Among the tested adsorbents, leaf-based materials (raw or activated forms) have received increased attention, mainly because they are cheap, available in large quantities in almost all regions of the world, required only a few stages of preparation, and in many cases not have another use. In addition, the use of leaf-based materials as sorbent materials for the sorption of dyes from aquatic effluents can significantly reduce the amount of waste produced, in agreement with the principles of circular economy.

On the other hand, the leaf-based materials of different trees contain a variety of inorganic and organic compounds, such as cellulose, hemicellulose, pectins, lignin, etc., [31]. These compounds have in their composition various functional groups, such as hydroxyl, carboxyl, carbonyl, amino, nitro [32], which can represent the binding sites in adsorption processes. The presence of such functional groups, which depending on experimental conditions may be dissociated or non-dissociated, makes leaf-based materials effective adsorbents to remove a variety of dyes (ionic or non-ionic) from aqueous media. In addition, the simple treatment of the raw leaf-based adsorbents with various chemical reagents, such as: (i) acids $\left(\mathrm{HCl}, \mathrm{HNO}_{3}, \mathrm{H}_{2} \mathrm{SO}_{4}\right.$, and $\mathrm{H}_{3} \mathrm{PO}_{4}$ ), (ii) bases $\left(\mathrm{NaOH}\right.$ and $\left.\mathrm{NaHCO}_{3}\right)$, (iii) oxidants $\left(\mathrm{KMnO}_{4}\right)$, (iv) inorganic salts $\left(\mathrm{NaCl}, \mathrm{CaCl}_{2}\right.$, and $\mathrm{MgCl}_{2}$ ), and organic reagents (formaldehyde, monosodium glutamate and anionic surfactant) [33] can significantly improve their adsorption capacity, and also their efficiency in dye removal processes.

There is only a recent review work available summarizing the published literature on the use of leaf-based adsorbents for heavy metals adsorption [34]. However, to the best of authors' knowledge, no other review is yet available discussing the application of leaf derived materials for the removal of dyes. This review is focused on the use of leafbased materials (raw or modified forms or activated carbons), as adsorbents for the removal of dyes from aqueous effluents, with applications in the wastewater treatment. This paper will be focused on the discussion of five key sections: (i) characteristics of leaf-based adsorbents, (ii) raw leaf-based adsorbents for dye removal, (iii) modified leafbased adsorbents for dye removal, (iv) equilibrium and kinetic aspects of dye adsorption on leaf-based adsorbents, and (v) possible applications for pilot scale and full scale systems.

\section{Analytical techniques used for adsorption studies}

Instrumental analysis plays a crucial role on adsorption studies, not only for the determination of dye concentration, but for the characterization of adsorbents prior and afterwards the interactions. This is a helpful asset in order to determine which features like structural, morphological, optical, and/or physicochemical, govern the adsorption capability, as well as for the elucidation of the involved mechanisms during the interactions of the dyes with the adsorbents. Additionally, the success and extent of chemical modification can be evaluated.

The analytical techniques that are usually reported for dye adsorption studies and the characterization of the samples include predominantly scanning electron microscopy (SEM), infrared spectroscopy or Fourier-transform infrared spectroscopy (FTIR), nuclear magnetic resonance (NMR), thermogravimetric analysis (TGA), differential scanning calorimetry (DSC) and spectrophotometry. The goals of the use of these techniques regarding dye removal using leaf-based adsorbents/ studies are summarized in Fig. 1.

Although each analytical technique provides specific information, the combination of different methods can bring light on the adsorption processes. Bellow, the contributions of each analytical technique on adsorption studies will be commented through examples.

\subsection{Scanning electron microscopy}

In many fields, such as chemistry, biology, geology, materials science, the study of the structural and morphological features of solids is highly important. One of the methods currently used to obtain this information is the Scanning electron microscopy (SEM). The SEM analysis provides a good insight regarding the surface and the shape of a solid. The electrons of the high energy focused beam used during the SEM analysis interact with atoms of the outer matrix of the material, generating different types of signals that contain information about the surface topography of the sample [35]. The resolution of this microscope can go above than $1 \mathrm{~nm}$. Regarding adsorption studies, SEM can be used in some cases to evaluate the size of the particles, the existence of cavities and channels [36], and in general the surface morphology of leaf-based adsorbents before and after dye adsorption [37-40]. It is not often, but under specific circumstances, morphological changes were reported after the interactions with different dyes [37]. Recently,
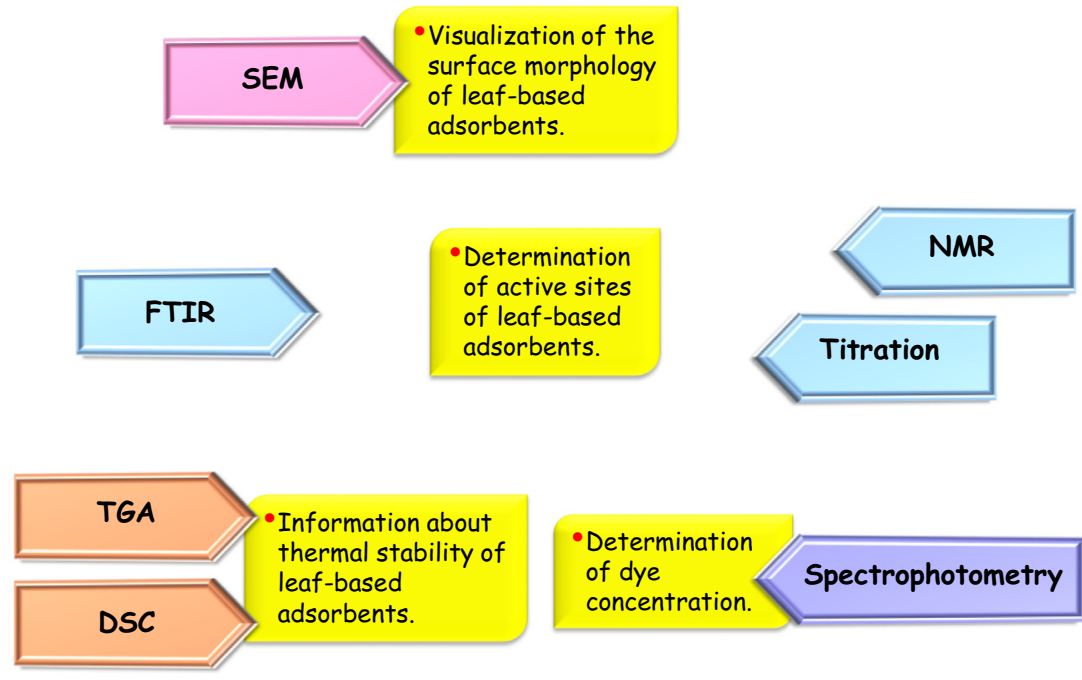

Fig. 1. Analytical techniques used for dye adsorption research. 
it has been reported the use of Salix babylonica leaves powder (SBLP) for the removal of methylene blue (MB) from aqueous solutions [38]. The SEM analysis of SBLP revealed that the adsorbent surface has numerous irregular cavities, which could be useful for the MB removal. The surface of exhausted/used SBLP was found markedly different compared to the initial one. The MB dye-loaded SBLP surface was smother in comparison with the initial sample and the absence of well-defined cavities in this micrograph suggests that the adsorption of the MB dye on the adsorbent occurred.

Typically, an energy dispersive X-ray microanalyzer (EDX) is coupled to the scanning electron microscope. This detector is useful to correlate the morphological characteristics of the adsorbent with its chemical composition, besides to obtain a mapping of the elemental concentration profiles, and perform quantitative analysis [39,40]. Several works have reported the use of SEM-EDX as a useful technique in the adsorption of dyes on leaf-based adsorbents and derivatives [41-43]. For instance, Baruah et al. studied SEM images before and after adsorption of MB on Aegle marmelos leaves (AMP) [43]. Before $\mathrm{MB}$ adsorption, the surface of AMP was rough and heterogeneous. However, after dye adsorption the extent of roughness and structural heterogeneity was weakened, due to the retention of MB molecules on specific areas. Moreover, the EDX analysis showed differences between the adsorbents' surface composition before and after MB adsorption. Before adsorption $\mathrm{C}, \mathrm{O}, \mathrm{Na}, \mathrm{K}, \mathrm{Ca}, \mathrm{Mg}$ and Fe were detected on the surface of the AMP, an elemental composition which is in accordance with the biological matrix of leaves. The elemental analysis of the spend sample showed the presence of S, suggesting the retention of sulfhydryl groups of the MB molecule on AMP surface.

\subsection{Fourier transform infrared spectroscopy}

The infrared region covers radiation with wavenumbers between 12,800 and $10 \mathrm{~cm}^{-1}$ and it is often to divide the infrared spectrum into three regions (near, medium and far). Up to 1980, the IR instruments for the medium region were of dispersive type, with the presence of diffraction networks. These dispersive spectrometers measure simultaneously the intensity over a narrow range of wavelengths. However, after 1980, great changes occurred in the medium IR instrumentation, with the appearance of instruments of the type of Fourier Transform. This shows a significant advantage over a dispersive spectrometer, due to it measures intensity over a wide range of wavelengths at a time. The term Fourier-transform infrared spectroscopy (FTIR) was originated from a mathematical process called "Fourier transform", which is necessary to convert the raw data into the final spectrum [44].

Fourier transform infrared spectroscopy is a vibrational spectroscopic technique usually used to identify functional groups present in leaf-based adsorbents surface that could be possibly be involved during the interactions/adsorption of the dyes $[45,46]$. Zhu et al. investigated the presence of functional groups on the Water Bamboo Leaves (WBL) by FTIR spectroscopy [47]. As can be observed in Fig. 2(a), the strong and wide band with a maximum at $3400 \mathrm{~cm}^{-1}$ can be assigned to the vibrations of the hydroxyl group linked in cellulose and lignin molecules or by adsorbed water [48]. Stretching vibrations of $\mathrm{C}-\mathrm{H}$ and $\mathrm{C}-\mathrm{O}$ bonds were attributed to the bands at $2922 \mathrm{~cm}^{-1}$ and $1639 \mathrm{~cm}^{-1}$, respectively. The band around $1384 \mathrm{~cm}^{-1}$ was assigned to the bending vibration of $\mathrm{C}-\mathrm{H}$. The band at $1261 \mathrm{~cm}^{-1}$ was attributed to the $\mathrm{C}-\mathrm{O}$ stretching present in the lignin molecule of the adsorbent and the band at $1076 \mathrm{~cm}^{-1}$ was assigned to $\mathrm{C}-\mathrm{O}-\mathrm{C}$ stretching of cellulose present in WBL. Furthermore, Fig. 2(b) shows the spectra of MBloaded WBL. It can be observed a decrease on the signal intensity of some bands, and the displacement or disappearance of other bands. These differences could be related to a possible involvement of specific functional groups on the WBL surface during the adsorption process.

FTIR technique is very helpful in the determination of functional groups present in chemically modified leaf-based adsorbents. Lim et al. developed a chemically modified adsorbent of $\mathrm{NaOH}$-Artocarpus

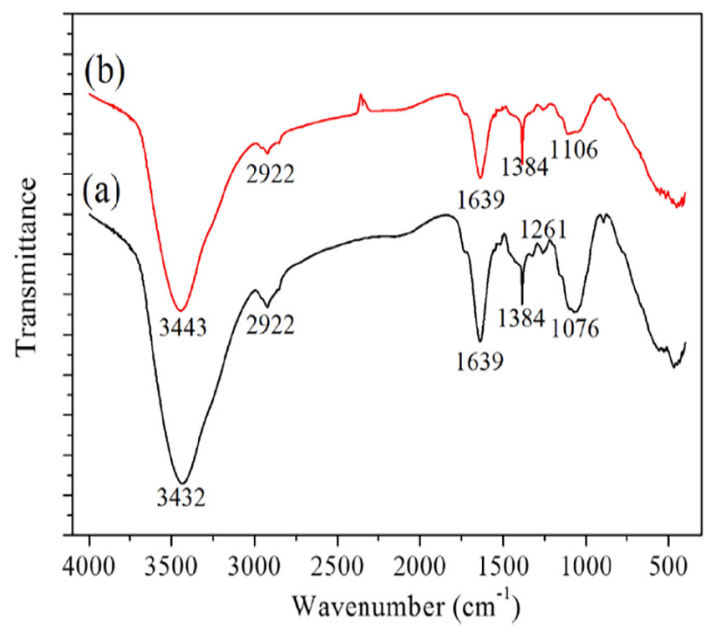

Fig. 2. FTIR spectra of Water Bamboo Leaves (a) before and (b) after methylene blue adsorption. Reproduced from [47] with kind permission of Springer.

odoratissimus leaves to remove cationic methyl violet 2B dye from aqueous solutions [49]. The spectra obtained for Artocarpus odoratissimus leaves and $\mathrm{NaOH}$-Artocarpus odoratissimus leaves showed a band at $3350 \mathrm{~cm}^{-1}$, which is characteristic of stretching vibrations of hydroxyl and amino groups of the surface adsorbents. A band at $1726 \mathrm{~cm}^{-1}$ is also present in both spectra and it was assigned to the presence of $\mathrm{C}=\mathrm{O}$ groups. The spectra of $\mathrm{NaOH}$-modified leaves showed slight shifts of $\mathrm{C}=\mathrm{C}$ stretching and $\mathrm{OH}$ bending vibration modes from 1611 to $1607 \mathrm{~cm}^{-1}$ and 1443 to $1420 \mathrm{~cm}^{-1}$, respectively. The most evident change was observed by the shift of $\mathrm{C}-\mathrm{O}$ stretching vibrations from 1042 to $1032 \mathrm{~cm}^{-1}$. These changes are indicative of a successful $\mathrm{NaOH}$-chemical modification of the leaf-based adsorbent.

In other work, activated carbon nanotubes (CNT-A) were studied as adsorbents for methyl orange (MO) dye [50]. The FTIR spectrum of CNTA revealed the presence of chemical defects at the CNT-A surface, besides the presence of $-\mathrm{OH}\left(3409 \mathrm{~cm}^{-1}\right), \mathrm{C}=\mathrm{C}\left(1686 \mathrm{~cm}^{-1}\right), \mathrm{C}=\mathrm{O}$ $\left(1548 \mathrm{~cm}^{-1}\right)$, and $\mathrm{C}-\mathrm{O}\left(1066 \mathrm{~cm}^{-1}\right)$ functional groups. These functional groups can possibly act as anchoring sites for MO dyes. Regarding the spectrum of the MO molecule, signals at 1013, 1185, 1355, and $1590 \mathrm{~cm}^{-1}$ were assigned to $-\mathrm{S}=\mathrm{O},-\mathrm{CH}_{3}, \mathrm{C}-\mathrm{N}$, and $-\mathrm{N}=\mathrm{N}-$ groups, respectively. Finally, the FTIR spectrum of MO exhausted CNT-A exhibited two new bands at 795 and $895 \mathrm{~cm}^{-1}$ in the fingerprint region of the spectrum. It can observed that the intensities of the signals at 1548 and $1687 \mathrm{~cm}^{-1}$ were increased because of the $-\mathrm{N}=\mathrm{N}-$ attaching to the aromatic ring of the $\mathrm{MO}$, which indicates that the dye molecule is anchored on the surface of CNT-A during the adsorption process. Moreover, the signal's intensity at $3409 \mathrm{~cm}^{-1}$ is enhanced, indicating that interactions via the formation of hydrogen bonds may be involved in the adsorption of MO. The above mentioned study can act as an example of a complete study that allow us to observe clear differences between the spectrum of the adsorbent, the pure dye molecule (which is a key aspect in adsorption studies) and that exhausted CNT-A sample, evidencing the interactions that govern the adsorption processes.

\subsection{Nuclear magnetic resonance}

Nuclear magnetic resonance (NMR) has been also used to characterize leaf-based adsorbents. Silva et al. used NMR to characterize Zea mays leaf powder to adsorb pollutants from aqueous solutions [51]. In this work, it was exhibited the ${ }^{13} \mathrm{C}$ NMR spectra obtained through $\left[{ }^{1} \mathrm{H}\right]-{ }^{13} \mathrm{C}$ cross-polarization technique (upper trace) and with direct polarization of ${ }^{13} \mathrm{C}$ (lower trace). The analysis of spectra revealed the main resonance at $74.3 \mathrm{ppm}$, which is assigned to $\mathrm{C}-\mathrm{OH}$ in glucoside and thiamine. The shoulder at $62 \mathrm{ppm}$ and the resonance at $104.5 \mathrm{ppm}$ can be correlated with $\mathrm{C}-\mathrm{OH}$ carbons of the glucoside. The resonances at $174 \mathrm{ppm}$ and 
Table 1

Maximum wavelengths usually used for the spectrophotometric analysis of some dyes.

\begin{tabular}{|c|c|c|c|c|}
\hline Dye & Molecular formula & Molecular structure & $\begin{array}{l}\lambda_{\max } \\
(\mathrm{nm})\end{array}$ & Reference \\
\hline Acid Blue 25 & $\mathrm{C}_{20} \mathrm{H}_{13} \mathrm{~N}_{2} \mathrm{NaO}_{5} \mathrm{~S}$ & & 655 & [59] \\
\hline $\begin{array}{l}\text { Acid Blue } \\
113\end{array}$ & $\mathrm{C}_{32} \mathrm{H}_{21} \mathrm{~N}_{5} \mathrm{Na}_{2} \mathrm{O}_{6} \mathrm{~S}_{2}$ & & 566 & {$[60]$} \\
\hline $\begin{array}{l}\text { Acid Green } \\
25\end{array}$ & $\mathrm{C}_{28} \mathrm{H}_{20} \mathrm{~N}_{2} \mathrm{Na}_{2} \mathrm{O}_{8} \mathrm{~S}_{2}$ & & 642 & [61] \\
\hline $\begin{array}{l}\text { Acid Orange } \\
52\end{array}$ & $\mathrm{C}_{14} \mathrm{H}_{14} \mathrm{~N}_{3} \mathrm{NaO}_{3} \mathrm{~S}$ & & 485 & [61] \\
\hline Acid red 27 & $\mathrm{C}_{20} \mathrm{H}_{11} \mathrm{~N}_{2} \mathrm{Na}_{3} \mathrm{O}_{10} \mathrm{~S}_{3}$ & & 522 & {$[62]$} \\
\hline $\begin{array}{l}\text { Acid violet } \\
17\end{array}$ & $\mathrm{C}_{41} \mathrm{H}_{44} \mathrm{~N}_{3} \mathrm{NaO}_{6} \mathrm{~S}_{2}$ & & 587 & [63] \\
\hline Basic Red 46 & $\mathrm{C}_{18} \mathrm{H}_{21} \mathrm{BrN}_{6}$ & & 530 & [64] \\
\hline $\begin{array}{c}\text { Brilliant } \\
\text { green }\end{array}$ & $\mathrm{C}_{27} \mathrm{H}_{33} \mathrm{~N}_{2} \cdot \mathrm{HO}_{4} \mathrm{~S}$ & & 624 & [65] \\
\hline Congo red & $\mathrm{C}_{32} \mathrm{H}_{22} \mathrm{~N}_{6} \mathrm{Na}_{2} \mathrm{O}_{6} \mathrm{~S}_{2}$ & & 490 & {$[66]$} \\
\hline $\begin{array}{l}\text { Crystal } \\
\text { violet }\end{array}$ & $\mathrm{C}_{25} \mathrm{~N}_{3} \mathrm{H}_{30} \mathrm{Cl}$ & & 580 & [67] \\
\hline $\begin{array}{c}\text { Eriochrome } \\
\text { Black T }\end{array}$ & $\mathrm{C}_{20} \mathrm{H}_{12} \mathrm{~N}_{3} \mathrm{O}_{7} \mathrm{SNa}$ & & 526 & [68] \\
\hline $\begin{array}{l}\text { Malachite } \\
\text { green }\end{array}$ & $\mathrm{C}_{23} \mathrm{H}_{25} \mathrm{ClN}_{2}$ & & 619 & [69] \\
\hline
\end{tabular}

Table 1 (continued)

\begin{tabular}{lllll}
\hline Dye & Molecular formula & Molecular structure & $\begin{array}{l}\lambda_{\max } \\
(\mathrm{nm})\end{array}$ & Reference \\
\hline $\begin{array}{l}\text { Methylene } \\
\text { blue }\end{array}$ & $\mathrm{C}_{16} \mathrm{H}_{18} \mathrm{ClN}_{3} \mathrm{~S}$ & 664 & {$[70]$} \\
$\begin{array}{c}\text { Rhodamine } \\
\text { B }\end{array}$ & $\mathrm{C}_{28} \mathrm{H}_{31} \mathrm{ClN}_{2} \mathrm{O}_{3}$ & &
\end{tabular}

$181 \mathrm{ppm}$ correspond to carbons at the - $\mathrm{COOH}$ groups in methionine and $\mathrm{N}=\mathrm{CN}$ in thiamine. The resonances observed between $146 \mathrm{ppm}$ and $153 \mathrm{ppm}$ can be assigned to $\mathrm{C}=\mathrm{C}$ and $\mathrm{N}=\mathrm{C}-\mathrm{S}$ in thiamine, respectively. The shoulder at $55.5 \mathrm{ppm}$ can be attributed to $\mathrm{CN}$ carbons in thiamine and methionine. Thus, the results obtained in both spectra for the adsorbent indicated that functional groups of thiamine and methionine can be present in Zea mays corn leaves.

\subsection{Titration}

Titration methods can be useful to identify specific surface functional groups of the adsorbents that have basic or acidic and ion-exchange properties [52-55]. Both Boehm and potentiometric titration methods have been applied in studies involving dye removal using leaf-based adsorbents. For instance, Rehman et al. studied the removal of brilliant green dye from water using Psidium guajava leaves as adsorbent and used the Boehm method as one tool to characterize the adsorbent [56]. In this work, carboxylic, phenolic and lactonic acid groups and total basic groups concentrations were estimated. The results showed that concentrations of acidic groups were higher than those of basic groups, which could be related with the subsequent adsorption of the dye.

\subsection{Thermal analysis}

Differential scanning calorimetry (DSC) and thermogravimetric analysis (TGA) are two of the most widely applied thermal analytical techniques in the characterization of adsorbents. In the TGA, the mass of the sample is measured as function of temperature increase during a period of time. Results from this analysis provide information related to physical and chemical phenomena, including physical adsorption, chemisorption, and thermal decomposition [57]. On the other hand, DSC gives information about the difference between heat flow required to increase the temperature of a sample and a reference as a function of temperature [57]. Coconus nucifera leaves have been used to produce carbon for the subsequent study of its adsorption behaviour [58]. The adsorbent was characterized by TGA, a very potent analytical technique. The thermogram obtained for leaves showed the thermal decomposition of the compounds present in the biological matrix, including cellulose, hemicellulose and lignin. Leaves exhibited three degradation steps: a) an initial weight loss below $110{ }^{\circ} \mathrm{C}$ that is related with water molecules loss; b) a $50 \%$ weight loss between 200 and $400{ }^{\circ} \mathrm{C}$ that is associated to the decomposition of cellulose and hemicellulose; and c) around $35 \%$ of the weight loss between 400 and $550{ }^{\circ} \mathrm{C}$ that may be attributed to the decomposition of lignin.

\subsection{Spectrophotometry}

UV-visible spectrophotometry is a suitable analytical technique for the determination of dyes concentration by recording the absorbance at specific wavelengths, normally the one with the highest intensity $\left(\lambda_{\max }\right)$. Table 1 shows the $\lambda_{\max }$ usually used for the spectrophotometric 
analysis of some dyes. The simplicity of this technique besides its low cost allows its use in several research laboratories.

\section{Leaf-based materials for dyes adsorption}

In this section, leaf-derived adsorbents were categorized in three groups: a) raw leaves, b) carbonaceous materials (such as activated carbons), and c) modified leaves.

Raw leaves are leaves that were used directly or subjected to washing several times using tap, distilled or deionized water in order to remove dust, dirt and other impurities. These samples were ground to powdery form and sieved to obtain the desirable particle size.

Activated carbon is the generic term used to describe a family of amorphous carbonaceous adsorbents with a highly crystalline form and well developed internal pore structure [72]. There are two different processes for the preparation of activated carbon: physical and chemical activation. The physical activation process involves the carbonization of the raw material under inert atmosphere, followed by activation at higher temperature in the presence of oxidizing gases [73]. Chemical activation, on the other hand, involves the carbonization of the precursor in the presence of chemical agents [74]. Some examples of chemical agents are zinc chloride $\left(\mathrm{ZnCl}_{2}\right)$, phosphoric acid $\left(\mathrm{H}_{3} \mathrm{PO}_{4}\right)$, sulphuric acid $\left(\mathrm{H}_{2} \mathrm{SO}_{4}\right)$, potassium hydroxide $(\mathrm{KOH})$ and sodium hydroxide $(\mathrm{NaOH})[75]$. ACs have porous structures and large surface areas (ranging from 500 to $2000 \mathrm{~m}^{2} / \mathrm{g}$ [76]). They are widely applied due to their excellent adsorption properties to remove volatile organic compounds and other contaminants from liquid and gaseous streams [77]. AC surface consists of basal planes, heterogeneous superficial groups (mainly oxygen containing functional groups such as hydroxyl, carboxyl, phenol, lactone, lactol, and quinone), and inorganic ash [78]. However, among all the sorbent materials proposed, activated carbon is the most popular for the removal of pollutants from wastewater [79]. Compare to other expensive treatment options, the utilization of ACs is an ideal alternative because they are effective in adsorbing wide range of dyes from wastewater [80]. Often, their application in wastewater treatment is sometimes restricted due to their high cost, low selectivity and regeneration factors [81].

The surface of the adsorbents can be modified via physical or chemical methods, before application for pollutants removal, the modifications are done primarily to improve the adsorption capacity of adsorbents [82]. Modified leaves adsorbents include materials (raw or carbonaceous) that are subjected to chemical surface modification using different chemical agents such as: acids, bases etc. After chemical modification, such adsorbent posess higher number of active binding sites, improved ion exchange properties and new functional groups that favors pollutant removal [83]. However, the cost implication of the modification process should be carefully considered to minimize the expenses incurred [84].

\subsection{Raw leaves for the adsorption of textile dyes}

The major parts of plant leaves are composed of cellulose, hemicellulose, pectins and lignin etc. [31], which contain various types of functional groups such as carboxyl, hydroxyl, carbonyl, amino, nitro [85] and hence can interact with functional groups of dyes. This interaction in turn leads to binding of dyes with leaves biomass [86-88]. Various studies have been carried out for the removal of different classes of dyes using leaf based biomass as adsorbent and very promising results have been obtained. For instance, Posidonia oceanica (L.) leaves were used for the removal of MB dye from aqueous solution in a fixed-bed column as a function of bed heights and flow rates (Table 2). Maximum dye adsorption was achieved at a flow rate of $7.28 \mathrm{~mL} / \mathrm{min}$ and bed height of $9 \mathrm{~cm}$. Both Thomas and Artificial Neural Network (ANN) models were employed for bed efficiency evaluation and Thomas model was revealed to fit the investigational data most. At optimum conditions, $P$. oceanica leaves showed a MB adsorption capacity (q, mg/g) of $482.6 \mathrm{mg} / \mathrm{g}$ [89].
Similarly, MB dye adsorption was also studied using Azadirachta indica leaves [90]. Various operational parameters were optimized for maximum dye adsorption such as adsorbent dosage, dye concentration, solution temperature and $\mathrm{pH}$. At optimum conditions (adsorbent dosage: $2 \mathrm{~g}$, dye concentration: $25 \mathrm{mg} / \mathrm{L}$, Temperature: $300 \mathrm{~K}$ ), $93 \%$ of MB dye was removed by the biosorbent. MB dye adsorption process was best described by Freundlich isotherm model, it also followed pseudo-first order kinetics with a rate constant of $3.73 \times 10^{-3} / \mathrm{min}$. Leaves of Posidonia oceanica (L.) Delile were also studied as adsorbent for MB dye adsorption [91]. The process variables such as $\mathrm{pH}$, adsorbent dosage, temperature and initial dye concentration were investigated using ANN model at optimum conditions of $\mathrm{pH} 6$, adsorbent dosage of $0.3 \mathrm{~g}$, temperature of $303 \mathrm{~K}$ and $50 \mathrm{mg} / \mathrm{L}$ initial dye concentration, up to 33\% MB dye adsorption was achieved. The MB dye adsorption onto $P$. oceanica leaves powder followed Langmuir isotherm with pseudo-second order kinetics model [91]. Moreover, the MB dye adsorption onto A. indica and P. oceanica leaves biomass was found very promising due to the strong interaction of the dye with functional groups present in the leaves. Hence, the use of $A$. indica and $P$. oceanica leaves biomass was strongly recommended for MB dye removal from textile wastewater.

Moreover, the fallen phoenix tree leaves showed very promising results for removal of MB dye from aqueous solution as a function of leaf dose, contact time, solution $\mathrm{pH}$, salt concentration and initial MB dye concentration. The adsorbent dose play a prominent role in MB dye adsorption, whereas medium $\mathrm{pH}$ did not affect the MB dye adsorption, while dye and salt concentration decreased the adsorption capacity of MB dye. The experimental data obtained from the dye adsorption process fitted Langmuir isotherm most with a maximum monolayer adsorption capacities of 80.9, 83.8, 89.7 (mg/g) observed at 295, 309 and $323 \mathrm{~K}$, respectively [92]. Similarly, Platanus orientalis leaves powder (POLP) was used for MB dye adsorption as a function of initial dye concentration, contact time, temperature and medium $\mathrm{pH}$. The adsorption data followed Langmuir isotherm with pseudo-second order kinetics. The POLP sorption capability was found to be $114.94 \mathrm{mg} / \mathrm{g}$. POLP also showed a good recycling ability. In view of the promising POLP adsorption capacity, it could possibly be utilized for the removal of dyes from textile effluents. In another study, Bauhinia purpurea L. leaves were used for elimination of MB dye. Process variables significantly affected the MB dye adsorption and the MB dye adsorption was found to be optimum at $\mathrm{pH} 10$ with agitation period of $20 \mathrm{~min}$ to equilibrate. Freundlich isotherm and pseudo-second order kinetic models were found to describe the MB dye adsorption data onto $B$. purpurea leaves powder most. The highest adsorption capability of $B$. purpurea was $78.37 \mathrm{mg} / \mathrm{g}$ at initial MB dye concentration of $35.34 \mathrm{mg} / \mathrm{L}$. Optimum adsorption were obtained at $\mathrm{pH} 10.48$, adsorbent dosage of $0.085 \mathrm{~g}$ at 31.8 ${ }^{\circ} \mathrm{C}$, respectively [93]. Similarly, the process variables effect on MB dye adsorption onto gulmohar ( $D$. regia) leaf powder was also studied [94]. Results revealed that temperature, adsorbent dosage, dye concentration, particle dimension, stirring rate and acidity of the medium considerably affected the MB dye adsorption onto the biosorbent. The dye adsorption experimental data correlated most with pseudo-secondorder kinetic model. Langmuir isotherm model fitted most with monolayer capability of $186.22 \mathrm{mg} / \mathrm{g}$. The optimum adsorption of MB dye was observed at higher $\mathrm{pH}$, initial dye concentration and a corresponding low adsorbent dosage. Gulmohar plant leaf powder showed promising efficiency for MB dye adsorption and in view of low cost, the use of this biomass was suggested for the remediation of dyes from textile wastewater [94]. Furthermore, Weng et al. [95] studied the efficiency of pineapple leaf powder (PLP) for the adsorption of MB dye. Maximum dye removal $\left(8.88 \times 10^{-4} \mathrm{~mol} / \mathrm{g}\right)$ was at high $\mathrm{pH}$, low temperature and low ionic strength. Dye adsorption kinetics was well described by Langmuir isotherm model with a pseudo-second order kinetic and intraparticle diffusion models. At low temperature and higher $\mathrm{pH}$, a highest removal ability of $9.28 \times 10^{-4} \mathrm{~mol} / \mathrm{g}$ was achieved. Results revealed that low cost PLP might successfully be used for the remediation of dyes from textile wastewater [95]. Fallen coconut leaves was also tested 
Table 2

Adsorption capacity of different plant leaves based adsorbent for the adsorption of different classes of dye.

\begin{tabular}{|c|c|c|c|c|c|c|}
\hline Leaves & Dye & Initial conditions & Maximum adsorption capacity (mg/g) & Isotherm model & Kinetic model & References \\
\hline Posidonia oceanica (L.) & MB & $\begin{array}{l}\text { Initial concentration: } 100 \mathrm{mg} / \mathrm{L} \text { ( fixed), } \\
\text { Contact time: } 0-1100 \mathrm{~min} \text {, } \\
\text { Flow rates: } 3.64-7.28 \mathrm{~mL} / \mathrm{min} \text {, } \\
\text { Bed height } 3-9 \mathrm{~cm}\end{array}$ & 482.6 & Th & NA & [89] \\
\hline Posidonia oceanica (L.) & MV & $\begin{array}{l}\text { Initial concentration: } 10-300 \mathrm{mg} / \mathrm{L} \text {, } \\
\text { Initial } \mathrm{pH} \text { : } 3-11 \text {, } \\
\text { Adsorbent dosage: } 0.025-1.000 \mathrm{~g} / 30 \mathrm{~mL} \text {, } \\
\text { Agitation speed: } 80-200 \mathrm{rpm} \text {, } \\
\text { Temperature: } 293-318 \mathrm{~K} \\
\text { Contact time: } 0-180 \mathrm{~min}\end{array}$ & 119.05 & $\mathrm{~L}$ & PSO & [97] \\
\hline Neem tree & BG & $\begin{array}{l}\text { Initial concentration: } 10-50 \mathrm{mg} / \mathrm{L} \text {, } \\
\text { Initial pH: } 6.5 \text { (not adjusted), } \\
\text { Adsorbent dosage: } 0.13-0.63 \mathrm{~g} / \mathrm{L} \text {, } \\
\text { Temperature: } 300-323 \mathrm{~K} \text {, } \\
\text { Contact time: } 60-300 \mathrm{~min}\end{array}$ & $98^{\mathrm{a}}$ & $\mathrm{L}$ & PFO & [103] \\
\hline Azadirachta indica & $\mathrm{MB}$ & $\begin{array}{l}\text { Initial concentration: } 25-70 \text {, } \\
\text { Initial pH: } 2-10 \text {, } \\
\text { Adsorbent dosage: } 2-10 \mathrm{~g} / \mathrm{L} \text {, } \\
\text { Temperature: } 300-340 \mathrm{~K} \text {, } \\
\text { Contact time: } 60-300 \mathrm{~min}\end{array}$ & 8.76 & $\mathrm{~F}$ & PFO & {$[90]$} \\
\hline Posidonia oceanica (L.) Delile & MB & $\begin{array}{l}\text { Initial concentration: } 10-100 \mathrm{mg} / \mathrm{L} \text {, } \\
\text { Initial pH: } 3-9 \text {, } \\
\text { Adsorbent dosage: } 0.05-1.0 \mathrm{~g} / 30 \mathrm{~mL} \text {, } \\
\text { Temperature: } 293-323 \mathrm{~K}, \\
\text { Contact time: } 5-180 \mathrm{~min}\end{array}$ & 10.06 & $\mathrm{~L}$ & PSO & [91] \\
\hline Paulownia tomentosa Steud. & A052 & $\begin{array}{l}\text { Initial concentration: } 20-100 \mathrm{mg} / \mathrm{L} \text {, } \\
\text { Initial } \mathrm{pH}: 2-10 \text {, } \\
\text { Adsorbent dosage: } 0.1-0.5 \mathrm{~g} / \mathrm{L} \text {, } \\
\text { Temperature: } 25-45^{\circ} \mathrm{C} \text {, } \\
\text { Contact time: } 0-180 \mathrm{~min} \text {, } \\
\text { Particle size: } 63-500 \mu \mathrm{m}\end{array}$ & 10.5 & $\mathrm{~L}, \mathrm{~T}$ & PSO & [99] \\
\hline Pine tree & BR46 & $\begin{array}{l}\text { Initial concentration: } 20-100 \mathrm{mg} / \mathrm{L} \text {, } \\
\text { Initial } \mathrm{pH} \text { : } 2-10 \text {, } \\
\text { Adsorbent dosage: } 1-6 \mathrm{~g} / \mathrm{L} \text {, } \\
\text { Temperature: } 25-45^{\circ} \mathrm{C} \text {, } \\
\text { Contact time: } 0-120 \mathrm{~min} \text {, } \\
\text { Particle size: } 63-500 \mu \mathrm{m}\end{array}$ & 71.94 & $\mathrm{~L}$ & PSO & [101] \\
\hline Princess tree & BR46 & $\begin{array}{l}\text { Initial concentration: } 20-100 \mathrm{mg} / \mathrm{L} \text {, } \\
\text { Initial } \mathrm{pH} \text { : } 2-10 \text {, } \\
\text { Adsorbent dosage: } 1-5 \mathrm{~g} / \mathrm{L} \text {, } \\
\text { Agitation speed: Temperature: } 25-45^{\circ} \mathrm{C} \text {, } \\
\text { Contact time: } 0-90 \mathrm{~min} \text {, } \\
\text { Particle size: } 63-500 \mu \mathrm{m}\end{array}$ & 43.10 & $\mathrm{~L}$ & PSO & [102] \\
\hline Eichhornia crassipes & Amaranth & $\begin{array}{l}\text { Initial concentration: } 50 \mathrm{mg} / \mathrm{L} \text {, } \\
\text { Initial pH: } 2.0 \text {, } \\
\text { Adsorbent dosage: } 1 \mathrm{~g} / \mathrm{L} \text {, } \\
\text { Agitation speed: } 120 \mathrm{rpm} \\
\text { Temperature: } 25^{\circ} \mathrm{C} \text {, } \\
\text { Contact time: } 0-48 \mathrm{~h} \text {, } \\
\text { Particle size: } 0.3-0.5 \mathrm{~mm}\end{array}$ & 43.1 & NA & PSO & {$[86]$} \\
\hline Hyacinth leaves & AR27 & $\begin{array}{l}\text { Initial concentration: } 10-500 \mathrm{mg} / \mathrm{L} \text {, } \\
\text { Initial pH: } 1.5-7.0, \\
\text { Adsorbent dosage: } 1 \mathrm{~g} / \mathrm{L} \text {, } \\
\text { Agitation speed: } 140 \mathrm{rpm} \text {, } \\
\text { Temperature: } 18-50{ }^{\circ} \mathrm{C} \text {, } \\
\text { Contact time: } 0-72 \mathrm{~h} \text {, } \\
\text { Particle size: } 0.15-0.18 \mathrm{~mm} \text { to } 1.68-2.0 \mathrm{~mm}\end{array}$ & 70.61 & $\mathrm{~L}$ & PSO & {$[100]$} \\
\hline Saraca asoca & MB & $\begin{array}{l}\text { Initial concentration: } 10-50 \mathrm{mg} / \mathrm{L} \text {, } \\
\text { Initial pH: } 2-7 \text {, } \\
\text { Adsorbent dosage: } 0.5-3 \mathrm{~g} / \mathrm{L} \text {, } \\
\text { Agitation speed: } 200 \mathrm{rpm} \text {, } \\
\text { Temperature: } 303-323 \mathrm{~K} \text {, } \\
\text { Contact time: } 0-50 \mathrm{~min} \text {, } \\
\text { Particle size: } 100-150 \mu \mathrm{m} \text {, } \\
\text { Ionic strength: } \mathrm{NaCl} 0-0.1 \mathrm{~mol} / \mathrm{L}\end{array}$ & 90.9 & $\mathrm{~F}$ & PSO & [87] \\
\hline Saraca asoca & MG & $\begin{array}{l}\text { Initial concentration: } 10-50 \mathrm{mg} / \mathrm{L} \text {, } \\
\text { Initial pH: } 2-7 \text {, } \\
\text { Adsorbent dosage: } 0.5-3 \mathrm{~g} / \mathrm{L} \text {, } \\
\text { Agitation speed: } 200 \mathrm{rpm} \text {, } \\
\text { Temperature: } 303-323 \mathrm{~K} \text {, } \\
\text { Contact time: } 0-50 \mathrm{~min} \text {, } \\
\text { Particle size: } 100-150 \mu \mathrm{m} \text {, } \\
\text { Ionic strength: } \mathrm{NaCl} 0-0.1 \mathrm{~mol} / \mathrm{L}\end{array}$ & 83.3 & $\mathrm{~F}$ & PSO & [87] \\
\hline Saraca asoca & RhB & $\begin{array}{l}\text { Initial concentration: } 10-50 \mathrm{mg} / \mathrm{L} \text {, } \\
\text { Initial } \mathrm{pH} \text { : } 2-7 \text {, }\end{array}$ & 66.6 & $\mathrm{~F}$ & PSO & [87] \\
\hline
\end{tabular}


Table 2 (continued)

\begin{tabular}{|c|c|c|c|c|c|c|}
\hline Leaves & Dye & Initial conditions & Maximum adsorption capacity (mg/g) & Isotherm model & Kinetic model & References \\
\hline Saraca asoca & BG & $\begin{array}{l}\text { Adsorbent dosage: } 0.5-3 \mathrm{~g} / \mathrm{L} \text {, } \\
\text { Agitation speed: } 200 \mathrm{rpm} \text {, } \\
\text { Temperature: } 303-323 \mathrm{~K} \text {, } \\
\text { Contact time: } 0-50 \mathrm{~min} \text {, } \\
\text { Particle size: } 100-150 \mu \mathrm{m} \text {, } \\
\text { Ionic strength: } \mathrm{NaCl} 0-0.1 \mathrm{~mol} / \mathrm{L} \\
\text { Initial concentration: } 10-50 \mathrm{mg} / \mathrm{L} \text {, } \\
\text { Initial pH: } 2-7 \text {, } \\
\text { Adsorbent dosage: } 0.5-3 \mathrm{~g} / \mathrm{L} \text {, } \\
\text { Agitation speed: } 200 \mathrm{rpm} \text {, } \\
\text { Temperature: } 303-323 \mathrm{~K} \text {, } \\
\text { Contact time: } 0-50 \mathrm{~min} \text {, } \\
\text { Particle size: } 100-150 \mu \mathrm{m}, \\
\text { Ionic strength: } \mathrm{NaCl} 0-0.1 \mathrm{~mol} / \mathrm{L}\end{array}$ & 125 & $\mathrm{~F}$ & PSO & [87] \\
\hline Fallen phoenix tree & MB & $\begin{array}{l}\text { Initial concentration: } 30-180 \mathrm{mg} / \mathrm{L} \text {, } \\
\text { Initial pH: } 2.5-10 \text {, } \\
\text { Adsorbent dosage: } 1-8 \mathrm{~g} / \mathrm{L} \text {, } \\
\text { Agitation speed: } 100 \mathrm{rpm} \text {, } \\
\text { Temperature: } 295-323 \mathrm{~K} \text {, } \\
\text { Contact time: } 0-200 \mathrm{~min} \text {, } \\
\text { Ionic strength: } \mathrm{NaCl} \text { or } \mathrm{CaCl}_{2} 0-0.2 \mathrm{~mol} / \mathrm{L}\end{array}$ & 80.9 & $\mathrm{~L}$ & NA & {$[92]$} \\
\hline Bauhinia purpurea & $\mathrm{CR}$ & $\begin{array}{l}\text { Initial concentration: } 20-100 \mathrm{mg} / \mathrm{L} \text {, } \\
\text { Initial pH: } 2-9 \text {, } \\
\text { Adsorbent dosage: } 0.1-0.5 \mathrm{~g} / 50 \mathrm{~mL} \text {, } \\
\text { Temperature: } 30 \pm 1{ }^{\circ} \mathrm{C} \text {, } \\
\text { Contact time: } 1-55 \mathrm{~min} \text {, } \\
\text { Particle size: } 81-162.3 \mu \mathrm{m}\end{array}$ & $84^{\mathrm{a}}$ & $\mathrm{L}$ & PSO & [104] \\
\hline Cicca acida & BR5B & $\begin{array}{l}\text { Initial concentration: } 10-100 \mathrm{mg} / \mathrm{L} \text {, } \\
\text { Initial pH: } 1-6 \text {, } \\
\text { Adsorbent dosage: } 0.01-0.06 \mathrm{~g} / 100 \mathrm{~mL} \text {, } \\
\text { Agitation speed: } 180 \mathrm{rpm} \\
\text { Temperature: } 25-35^{\circ} \mathrm{C} \text {, } \\
\text { Contact time: } 0-240 \mathrm{~min}\end{array}$ & 196.08 & $\mathrm{~L}$ & PSO & [105] \\
\hline Platanus orientalis & $\mathrm{MB}$ & $\begin{array}{l}\text { Initial concentration: } 20-80 \mathrm{mg} / \mathrm{L} \text {, } \\
\text { Initial pH: } 2-12 \text {, } \\
\text { Adsorbent dosage: } 80 \mathrm{mg} / 50 \mathrm{~mL} \text {, } \\
\text { Agitation speed: } 200 \mathrm{rpm} \\
\text { Temperature: } 25-60^{\circ} \mathrm{C} \text {, } \\
\text { Contact time: } 0-70 \mathrm{~min}\end{array}$ & 114.94 & $\mathrm{~L}$ & PSO & {$[106]$} \\
\hline Bauhinia purpurea L. & $\mathrm{MB}$ & $\begin{array}{l}\text { Initial concentration: } 20-100 \mathrm{mg} / \mathrm{L} \text {, } \\
\text { Initial pH: } 2-12 \text {, } \\
\text { Adsorbent dosage: } 0.02 \text { to } 0.1 \mathrm{~g} / 30 \mathrm{~mL} \text {, } \\
\text { Temperature: } 30-50{ }^{\circ} \mathrm{C} \text {, } \\
\text { Contact time: } 0-60 \mathrm{~min}\end{array}$ & 78.37 & $\mathrm{~F}$ & PSO & [93] \\
\hline Delonix regia & $\mathrm{MB}$ & $\begin{array}{l}\text { Initial concentration: } 50-200 \mathrm{mg} / \mathrm{L} \text {, } \\
\text { Initial pH: } 2-9 \text {, } \\
\text { Adsorbent dosage: } 0.5-2.5 \mathrm{~g} / \mathrm{L} \text {, } \\
\text { Agitation speed: } 50-200 \mathrm{rpm} \text {, } \\
\text { Temperature: } 303-323 \mathrm{~K}, \\
\text { Contact time: } 24 \mathrm{~h} \text {, } \\
\text { Particle size: } 40-530 \mu \mathrm{m}\end{array}$ & 186.22 & $\mathrm{~L}$ & PSO & [94] \\
\hline Artocarpus heterophyllus & $\mathrm{CV}$ & $\begin{array}{l}\text { Initial concentration: } 20-100 \mathrm{mg} / \mathrm{L} \text {, } \\
\text { Initial pH: } 3-8.0 \text {, } \\
\text { Adsorbent dosage: } 1 \mathrm{~g} / 0.1 \mathrm{~L} \text {, } \\
\text { Agitation speed: } 150 \mathrm{rpm} \text {, } \\
\text { Temperature: } 293-313 \mathrm{~K} \text {, } \\
\text { Contact time: } 10-180 \mathrm{~min} \text {, } \\
\text { Particle size: }(100-125 \mu \mathrm{m} \text { (fixed) }\end{array}$ & 43.39 & L, Th & PSO & [98] \\
\hline Pineapple & $\mathrm{MB}$ & $\begin{array}{l}\text { Initial concentration: } 5 \times 10^{-6}-4 \times 10^{-5} \mathrm{M} \text {, } \\
\text { Initial pH: } 3.5-9.5 \text {, } \\
\text { Adsorbent dosage: } 0.5 \mathrm{~g} / \mathrm{L} \text {, } \\
\text { Agitation speed: } 200-500 \mathrm{rpm} \text {, } \\
\text { Temperature: } 4-54{ }^{\circ} \mathrm{C} \text {, } \\
\text { Contact time: } 0-80 \mathrm{~min} \text {, } \\
\text { Ionic strength: } \mathrm{NaNO}_{3} 5 \times 10^{-3}-7 \times 10^{-2} \mathrm{M}\end{array}$ & $8.88 \times 10^{-4 b}$ & $\mathrm{~L}$ & PSO & [95] \\
\hline Fallen coconut leaves & MB & $\begin{array}{l}\text { Initial concentration: } 10-50 \mathrm{mg} / \mathrm{L} \text {, } \\
\text { Initial } \mathrm{pH} \text { : } 4-10 \text {, } \\
\text { Adsorbent dosage: } 0.5-1.5 \mathrm{~g} / \mathrm{L} \text {, } \\
\text { Agitation speed: } 120 \mathrm{rpm} \text {, } \\
\text { Temperature: } 27 \pm 2{ }^{\circ} \mathrm{C} \\
\text { Contact time: } 5-45 \mathrm{~min}, \\
\text { Particle size: } 150-212 \mu \mathrm{m} \text { (fixed) }\end{array}$ & 112.35 & $\mathrm{~L}$ & PSO & {$[96]$} \\
\hline
\end{tabular}

NA: Not available, $\mathrm{L}=$ Langmuir isotherm, $\mathrm{F}=$ Freundlich isotherm, Y-N = Yoon-Nelson, Th = Thomas, PFO = Pseudo-first order kinetic, PSO = Pseudo-second order kinetic. a \%.

b $\mathrm{mol} / \mathrm{g}$. 
for its efficiency in adsorbing MB dye [96]. Batch adsorption experiments were conducted at $\mathrm{pH}(4.0-10.0)$, contact time (5-45 min), initial concentration (10-50 mg/L) and adsorbent's dosage (0.5-1.5 g/L). Optimum conditions were found to be at $\mathrm{pH} 8.65,5 \mathrm{~min}$ of contact time, and $1.26 \mathrm{~g} / \mathrm{L}$ dosage.

Methyl violet (MV) dye was also remediated using plant leaf based adsorbents, i.e., MV adsorption was investigated using Posidonia oceanica (Linnaeus) Delile leaves [97]. The process variables significantly affected the MV dye adsorption. The process was best described with pseudo-second order kinetic model, the data fitted Langmuir model most among the isotherms used (Langmuir, Freundlich, and Dubinin-Radushkevich), with a greatest sorption capability of $119.05 \mathrm{mg} / \mathrm{g}$ at $45^{\circ} \mathrm{C}$. In comparison to various other plant based adsorbents, $P$. oceanica gave considerably higher efficiency for the adsorption of MV dye and could possibly be used as a lost cost adsorbent for the remediation of textile dyes wastewater [97]. Gupta al. [87] also revealed that ashoka plant leaf powder (APLP) served as efficient adsorbent for the elimination of MB dye from aqueous medium and at optimum conditions of the process, up to $90.9 \mathrm{mg} / \mathrm{g}$ dye was absorbed on to APLP. Similarly, crystal violet (CV) dye was removed using Artocarpus heterophyllus leaf powder (AHLP) at optimum conditions of process variables (contact time, $\mathrm{pH}$, initial dye concentration and temperature). The experimental data fitted best to the Langmuir isotherm and pseudo-second order kinetic models following a chemisorption mechanism. A maximum monolayer biosorption capacity of $43.39 \mathrm{mg} / \mathrm{g}$ was observed at $\mathrm{pH} 7.0$, initial dye concentration $50 \mathrm{mg} / \mathrm{L}, 293 \mathrm{~K}$ at 120 min contact time. The CV dye adsorption was also studied using fixed-bed column; adsorption increased linearly with increase in bed height and decreased with increase in flow rate. Thomas and BDST models agreed well with the experimental data. Results revealed that AHLP is an competent adsorbent for sorption of CV dye and can also be applied for other dyes from textile wastewater since biosorption capacity of AHLP was higher in comparison with other plant based biosorbents [98].

Acid orange 52 (A052) dye adsorption was promising with the usage of Paulownia tomentosa Steud leaves biomass, medium acidity, adsorbent dose, dye concentration, size of particle, temperature and exposure time significantly affected the dye adsorption. Temkin and Langmuir isotherms best fitted the dye adsorption data with pseudo-second order kinetic model with a highest sorption ability of $10.5 \mathrm{mg} / \mathrm{g}$. The results indicated that $P$. tomentosa leaves might be an efficient adsorbent for the removal of AO52 from wastewater [99].

In another study, Acid Red 27 (AR27) an anionic dye was adsorbed using hyacinth leaves [100]. The effects of process variables were observed and it was noted that AR27 removal was enhanced with contact time and initial dye concentration and decreased at low medium $\mathrm{pH}$. Langmuir isotherm with pseudo-second-order kinetic models fitted most with AR27 dye adsorption data onto hyacinth leaves powder. Maximum monolayer coverage was $70 \mathrm{mg} / \mathrm{g}$. Results revealed that hyacinth leaves has efficient adsorption capacity and is capable of remediating AR27 dye from wastewaters [100].

Basic Red 46 (BR46) dye also showed excellent interaction with another plant leaf based adsorbents. Pine tree leaves were investigated for the adsorption of BR46 dye as a function of various process variables and it was noticed that the dye adsorption data fitted the Langmuir isotherm most with pseudo-second order model describing the kinetic data most having a monolayer sorption capacity of $71.94 \mathrm{mg} / \mathrm{g}$. Results demonstrated that the pine tree leaves are efficient for BR46 dye adsorption and can also be used for other dye removal from textile wastewater [101]. Similarly, princess tree leaf (PTL) also showed promising efficiency for removal of BR46 dye from aqueous solution [102]. The process variables significantly affected the dye adsorption onto PTL and the dye adsorption equilibrium was achieved in $70 \mathrm{~min}$ at $\mathrm{pH}$ 8.0, high initial dye concentration, low (temperature, biosorbent dosage and particle size). The Langmuir maximum monolayer sorption capacity was $43.10 \mathrm{mg} / \mathrm{g}$ at $25^{\circ} \mathrm{C}$ and dye adsorption followed pseudo-second order kinetic model. Thus, PTL showed an excellent adsorption capacity and it is recommended as a cheap and easily available adsorbent for the elimination of other dyes from textile wastewater [102].

Brilliant Green (BG) adsorption was also investigated using plant leaves based adsorbent. Neem tree leaves were successfully used for BG dye adsorption [103]. Adsorbent doses, $\mathrm{pH}$ values and temperature showed variable effect on BG dye adsorption. Fitting the removal data to Langmuir and Freundlich isotherms and by computing equilibrium thermodynamic and kinetic parameters, the suitability of Neem tree leaves as adsorbent was very promising for BG dye adsorption. Similarly, Gupta al. [87] also revealed that ashoka plant leaf powder (APLP) was very efficient for the elimination of BG dye and at optimum conditions of process variables, up to $125 \mathrm{mg} / \mathrm{g}$ BG dye was absorbed on to APLP.

Various other textile dyes such as amaranth dye, MG, RhB, CR, BR5B have also been adsorbed using different plant leaves based adsorbents like Eichhornia crassipes, Bauhinia purpurea and Ciccaacida, i.e., amaranth dye biosorption on to the leaves of Eichhornia crassipes (water hyacinth) showed promising efficiency and up to $43.1 \mathrm{mg} / \mathrm{g}$ was adsorbed with a pseudo-second order kinetic model, it revealed the chemisorption nature of amaranth dye onto E. crassipes leaves. In view of excellent efficiency and low-cost, E. crassipes leaves could possibly be used for the remediation of polluted water with textile dyes [86]. Ashoka leaf powder (ALP) also showed interactive behaviour for rhodamine $\mathrm{B}(\mathrm{RhB})$, malachite green and brilliant green dye. Process variables were optimized for highest dye sorption and the sorption results obeyed Freundlich isotherm and pseudo-second order kinetic model. In view of promising efficiency to remove various dyes, the use of ALP was suggested for remediation of textile wastewater [87]. Similarly, Congo Red (CR) was successfully removed using on Bauhinia purpurea leaves. At optimized conditions, the CR dye removal was $84 \%$ using $0.1 \mathrm{~g}$ adsorbent dose, $20 \mathrm{mg} / \mathrm{L}$ of initial dye concentration for $40 \mathrm{~min}$ contact time at $\mathrm{pH}$ 6.0. The Langmuir isotherm best describes the $\mathrm{CR}$ dye sorption data with pseudo-second order kinetic model [104]. In another contribution, Brilliant Red 5B dye (BR5B) was removed using Cicca acida leaves. At optimum conditions (initial dye concentration of $100 \mathrm{mg} / \mathrm{L}, \quad \mathrm{pH} 2.0,30{ }^{\circ} \mathrm{C}$ ) maximum BR5B dye adsorption ( $196.08 \mathrm{mg} / \mathrm{g}$ ) was achieved onto $C$. acida leaves. Langmuir isotherm and pseudo-second order kinetic models best disclosed the BR5B dye adsorption data onto C. acida leaves [105].

The characterization studies revealed that the raw leaves have interactive properties with dyes due to different physico-chemical properties. FTIR analysis revealed that leave-based biomass possesses various functional groups, which are responsible for dye adsorption on the surface of adsorbent. A peak appeared at $3400 \mathrm{~cm}^{-1}$ was due to stretching vibration of $\mathrm{O}-\mathrm{H}$ bond in hydroxyl functional group, whereas band at 2934 and $1386 \mathrm{~cm}^{-1}$ are correlated with the stretching and bending vibrations of $\mathrm{C}-\mathrm{H}$ bond in methyl groups respectively and carbonyl group stretching was observed at $1736 \mathrm{~cm}^{-1}$. Similarly, a band recorded at $1603 \mathrm{~cm}^{-1}$ was assigned to $\mathrm{C}=\mathrm{C}$ group (bending vibration), whereas $1052 \mathrm{~cm}^{-1}$ band revealed the presence of $\mathrm{C}-\mathrm{O}$ functional group due to lignin structure of the ALP. The band appearing at $1250 \mathrm{~cm}^{-1}$ indicates $\mathrm{C}-\mathrm{O}$ (stretching vibration) due to phenols [87,107]. Similarly, the interaction of amaranth dye was correlated with functional groups present on the surface of biosorbent [86]. The FTIR spectra of native and dye loaded of E. crassipes leaves powder. Spectra clearly indicate the presence of various functional groups since a large number of bands are detected in native biomass, which revealed the presence of $-\mathrm{COOH}$, $-\mathrm{NH}_{2},-\mathrm{NH},-\mathrm{OH}$ functional groups due to proteins, fats and crude fibre in leaf biomass. The peaks at 3800-3500, 3000-2500 $\mathrm{cm}^{-1}$ $2930-2920 \mathrm{~cm}^{-1}, 1660 \mathrm{~cm}^{-1}, 1520 \mathrm{~cm}^{-1}, 1456-1410 \mathrm{~cm}^{-1}$, $1100 \mathrm{~cm}^{-1}, 1500-400 \mathrm{~cm}^{-1}$ are assigned to the $-\mathrm{OH},-\mathrm{NH}, \mathrm{C}-\mathrm{H}$ stretching vibration, $\mathrm{C}=\mathrm{O}$ stretching (amide I), $\mathrm{NH}$ (amide II), $\mathrm{C}-\mathrm{O}$, $\mathrm{C}-\mathrm{O}-\mathrm{C}$ groups and ionic interactions [87]. It was observed that the functional groups were shifted in dye loaded biomass. In loaded E. crassipes leaves powder, the peaks at the $3800-3500 \mathrm{~cm}^{-1}$, 
$3000-2500 \mathrm{~cm}^{-1}$ were correlated with change in hydrogen bonds due to dye interaction with $-\mathrm{OH}$ and $-\mathrm{NH}$ groups. Interestingly, the absorption characteristics bands due to amide I at $1650 \mathrm{~cm}^{-1}$ and amide II $\left(1540 \mathrm{~cm}^{-1}\right)$ were also shifted since dye interacted with these functional groups at these regions. The bands $1716 \mathrm{~cm}^{-1}, 1732 \mathrm{~cm}^{-1}$, and $1732 \mathrm{~cm}^{-1}$ were also shifted, which also revealed the interaction of functional groups of dye with that on the surface of the biosorbent. In loaded biomass the $-\mathrm{OH}$ peak was shifted from 1410 to $1433 \mathrm{~cm}^{-1}$ indicating the interaction of $\mathrm{C}-\mathrm{O}$ groups (due to proteins, cellulose, hemicelluloses and/or lignin) with dye molecule. E. crassipes leaves analysis revealed the presence of $33.34 \%$ protein (a base for amide groups) and FTIR analyses (native and dye loaded) indicated the interaction of these groups with amaranth dye molecule. It was also reported that due to the electrostatic attraction between the negatively charged sulfonic groups of dyes and positively charged amide groups a bond is developed between the amaranth dye and proteins of leaves. Moreover, the hydrophobic interaction between the dye molecule and the polypeptide backbone attract the positively charged groups of the proteins toward dye molecule $[86,108]$.

\subsection{Carbonaceous adsorbents (such as carbonized materials, activated carbons etc.) derived from leaves}

Activated carbon can be obtained from different parts of plant materials such as roots, stems, leaves, fruits etc. It is a very versatile substance which has several uses. It is used in air, water and gold purification, gas storage (methane and hydrogen storage), air filters in gas masks and respirators, filters in compressed air, decaffeination, gold purification, metal extraction, sewage treatment, medicine, teeth whitening, and many other applications. In the industry, it is used in metal finishing for purification of electroplating solutions $[74,75]$.

Activated carbon has a high surface area, this property made them very useful in special applications. Physically, activated carbon binds materials by van der Waals force or London dispersion force. The properties of AC could be physical or chemical depending on the precursor material and activation method used. They are prepared either directly from the starting material only (e.g. activated carbon from leaves) or combining the starting material with activating agents such as $\mathrm{HNO}_{3}$, $\mathrm{H}_{2} \mathrm{SO}_{4}, \mathrm{HCl}, \mathrm{NaOH}, \mathrm{ZnCl}_{2}$ etc. (modified activated carbon from leaves) $[75,76]$. This section emphasizes AC derived mainly from leaves.

Bello and Semire investigated the ability of Imperata cylindrica activated carbon (ICAC) for the removal of Congo red (CR) dye from aqueous solution. The maximum adsorption capacity was obtained at $\mathrm{pH} 3.0$ (tested pH range 3.0-10.0), while the equilibrium reached in 150 and 180 min of contact time at lowest and highest concentrations, respectively. Quantum chemical studies suggested that the protonation of aniline groups and minimal molecular size at planar geometry coupled with electrostatic interaction enhances the adsorption at low $\mathrm{pH}$. The dye uptake process obeyed the pseudo-second order kinetic expression $\left(R^{2} \geq 0.99\right)$ and was best described by the Langmuir isotherm $\left(R^{2} \geq\right.$ 0.98). The maximum monolayer capacity estimated to be $312.75 \mathrm{mg} / \mathrm{g}$. Their study revealed that ICAC was effective in removing CR dye from aqueous solutions [109].

Gunasekar and Ponnusami reported the carbonized senescent plant leaves powder for MB dye remediation from effluent [110]. The influences of different operational parameters were examined to ascertain the feasibility of the carbonized adsorbent for efficient removal of MB dye. Adsorption kinetics was in good agreement with PSO-model. The isotherm fitted Langmuir than the Freundlich model on the basis of higher $R^{2}$ and lower chi ${ }^{2}$ values. The maximum monolayer adsorption capacity obtained was $61.22 \mathrm{mg} / \mathrm{g}$. From the thermodynamic point of view, the $\Delta G^{0}, \Delta H^{0}$, and $\Delta S^{0}$ values for the MB-dye adsorption was feasible and spontaneous. Judging from the $\Delta H^{0}$ magnitude, the adsorption process was established to be endothermic in nature and physically controlled.

Similarly, Jawad et al. [111] used $\mathrm{H}_{2} \mathrm{SO}_{4}$ as an activating agent to prepare efficient activated carbon from coconut leaves. The authors also investigated the influences of different variables governing the adsorption of $\mathrm{MB}$ onto activated coconut leaves and found that $\mathrm{H}_{2} \mathrm{SO}_{4}$ activated carbon, a low-cost adsorbent was highly efficient for the removal of MB-dye from aqueous solution. Langmuir isotherm model showed a better fit with an increased adsorption capacity at different temperature and thus found to increase with increasing temperature from 126.9 at $303 \mathrm{~K}$ to 137.0 at $313 \mathrm{~K}$ and to $149.3 \mathrm{mg} / \mathrm{g}$ at $323 \mathrm{~K}$, respectively. The kinetics of MB-dye uptake agreed well with PSO-model at all temperature. Thermodynamic studies showed that $\Delta G^{0}, \Delta H^{0}$ and $\Delta S^{0}$ were favourably driven by the entropic factor which was also in agreement with a lower energy of activation of the system (i.e. $29.70 \mathrm{~kJ} / \mathrm{mol}$ ). It was therefore concluded that $\mathrm{H}_{2} \mathrm{SO}_{4}$-activated carbons from coconut leaf is a good precursor for AC preparation in the remediation $\mathrm{MB}$ dye from aqueous solution. Similarly, thermal carbonization via $\mathrm{FeCl}_{3-}$ activation was used to prepare activated carbon from coconut leaves [112]. The characterization studies were investigatedby bulk density, moisture content, ash content, $\mathrm{pH}_{p z c}$, iodine test, elemental (CHNS-O) analysis and other spectroscopic analyses. It was shown that $\mathrm{FeCl}_{3}$-activated carbon $(F A C)$ has an iodine number of $653.00 \mathrm{mg} / \mathrm{g}$. The effect of varying adsorbent dosage from 0.02 to $0.25 \mathrm{~g}, \mathrm{pH} 3-11$, initial dye concentrations from 30 to $350 \mathrm{mg} / \mathrm{L}$ and contact time from 1 to $180 \mathrm{~min}$ at $303 \mathrm{~K}$ were via batch experiments. They observed that PSO described the kinetic data best whereas; Langmuir isotherm established the adsorptive behaviour at equilibrium with maximum adsorption capacity $\left(\mathrm{q}_{\max }\right)$ of $66.00 \mathrm{mg} / \mathrm{g}$.

Ghosh and Bandyopadhyay [113] reported the removal of MB dye from aqueous solutions using citric acid as activating agent on carbonized bamboo leaves powder. Batch experiment which is a function of different operational variables was investigated. Among other seven isotherms investigated, Temkin was found most favourable and more so, the kinetic fitted well with PSO-model. Additional studies revealed that the rate at which intra-particle diffusion occurred was controlled by film diffusion. Thermodynamic studies showed the spontaneity and chemisorption nature of adsorption with negative $\Delta G^{0}$ decreasing from 33.70 to $25.43 \mathrm{~kJ} / \mathrm{mol}$; while $\Delta H^{0}$ and $\Delta S^{0}$ were $134.97 \mathrm{~kJ} / \mathrm{mol}$ and $554.51 \mathrm{~J} / \mathrm{mol} \mathrm{K}$ respectively. It was also observed that the energy of activation followed chemisorption mechanism ranging from 84.07 to $98.90 \mathrm{~kJ} / \mathrm{mol}$ determined from PSO rate constant as a function of temperature. The novelty accomplished in this study was higher percentage MB dye removal of $99.97 \%$ and maximum adsorption capacity of $725 \mathrm{mg} / \mathrm{g}$ respectively; with a final $\mathrm{pH}$ of solution falling within the safe discharge limits.

The potential of coconut leaf mesoporous-activated carbon prepared via microwave-induced $\mathrm{H}_{3} \mathrm{PO}_{4}$ activation using batch process for $\mathrm{MB}$ dye removal was investigated [114]. Experiments were carried as a function of contact time (0-360 min), adsorbent dose (0.2-10 g/L), initial concentration $(50-350 \mathrm{mg} / \mathrm{L})$ and initial $\mathrm{pH}(3-10)$. The optimum condition wereas follows: $\mathrm{pH}$ 5.0-6.0 and adsorbent dosage $2 \mathrm{~g} / \mathrm{L}$. The adsorption of MB dye onto CAC was found to be spontaneous and exothermic in nature.

Activated carbon from fallen coconut (Cocos nucifera) leaves were prepared by thermal carbonization using $\mathrm{KOH}$-activation method and used to remove MB dye [115]. Among examined parameters, the effect of $\mathrm{pH}$ was found to be negligible whereas the increase in temperature from 303 to $323 \mathrm{~K}$ affected the sorption process of MB dye onto activated carbon. $E_{\text {a }}$ was found to be $8.43 \mathrm{~kJ} / \mathrm{mol}$ suggesting physisorption mechanism. The adsorption of MB dye onto activated carbon was found to be both spontaneous and endothermic in nature.

Guava leaf activated carbon (GLAC) was prepared by [116] to remediate Congo red $(\mathrm{CR})$. Interpretation of activation process revealed introduction of additional surface functional groups on the carbon precursors evidenced in the spectra with broadening, reduction or disappearance of the peaks after the activation of the raw precursor leading to a production of entirely new carbon materials. More so, an increased in $\mathrm{C}-\mathrm{H}_{3} \mathrm{PO}_{4}$ reaction rate as a result of chemical activation, thus resulted 
Table 3

Carbonaceous adsorbents derived from leaves for dyes removal.

\begin{tabular}{|c|c|c|c|c|c|c|}
\hline Adsorbents & Dye & Initial conditions & Isotherm models & Kinetic models & $\begin{array}{l}\text { Maximum adsorption } \\
\text { capacity }(\mathrm{mg} / \mathrm{g})\end{array}$ & Refs. \\
\hline $\begin{array}{l}\text { Activated carbons obtained from } \\
\text { spent black tea leaves (soaked } \\
\text { in } \mathrm{H}_{2} \mathrm{SO}_{4} \text { at } 100{ }^{\circ} \mathrm{C} \text { ) followed by } \\
\text { the microwave treatment }\end{array}$ & EBT & $\begin{array}{l}\text { Initial concentration: } 10-400 \mathrm{mg} / \mathrm{L} \text {, } \\
\text { Initial pH: } 2-9 \text {, } \\
\text { Adsorbent dosage: } 0.25-5.0 \mathrm{~g} / \mathrm{L} \text {, } \\
\text { Temperature: } 25-60{ }^{\circ} \mathrm{C} \\
\text { Contact time: } 0-800 \mathrm{~min}\end{array}$ & $\mathrm{~L}$ & PSO & 242.72 & [117] \\
\hline $\begin{array}{l}\text { Carbons obtained senescent plant } \\
\text { leaves (Carbonized at } 500{ }^{\circ} \mathrm{C} \text { ) }\end{array}$ & $\mathrm{MB}$ & $\begin{array}{l}\text { Initial concentration: } 50-200 \mathrm{mg} / \mathrm{L} \text {, } \\
\text { Initial pH: } 2-9 \text {, } \\
\text { Adsorbent dosage: } 0.5-2 \mathrm{~g} / \mathrm{L} \text {, } \\
\text { Agitation speed: } 200 \mathrm{rpm} \text {, } \\
\text { Temperature: } 293-323 \mathrm{~K} \text {, } \\
\text { Contact time: } 0-150 \mathrm{~min} \text {, } \\
\text { Average particle size: } 90 \mu \mathrm{m}\end{array}$ & $\mathrm{L}$ & PSO & 61.22 & [110] \\
\hline $\begin{array}{l}\text { Activated carbons obtained } \\
\text { coconut leaves impregnated } \\
\text { with } \mathrm{H}_{2} \mathrm{SO}_{4}\end{array}$ & MB & $\begin{array}{l}\text { Initial concentration: } 30-400 \mathrm{mg} / \mathrm{L} \text {, } \\
\text { Initial pH: } 3-11 \text {, } \\
\text { Adsorbent dosage: } 0.2-2.5 \mathrm{~g} / \mathrm{L} \text {, } \\
\text { Agitation speed: } 110 \mathrm{rpm} \text {, } \\
\text { Temperature: } 303-323 \mathrm{~K} \text {, } \\
\text { Contact time: } 0-180 \mathrm{~min} \text {, } \\
\text { Particle size: } 150-212 \mu \mathrm{m} \text { (fixed) }\end{array}$ & $\mathrm{L}$ & & 149.3 & [111] \\
\hline $\begin{array}{l}\text { Citric acid treated carbonized } \\
\text { bamboo leaves powder } \\
\text { (carbonized at } 450{ }^{\circ} \mathrm{C} \text { ) }\end{array}$ & $\mathrm{MB}$ & $\begin{array}{l}\text { Initial concentration: } 100-400 \mathrm{mg} / \mathrm{L} \text {, } \\
\text { Initial pH: } 7.5 \text {, } \\
\text { Adsorbent dosage: } 0.2-1.4 \mathrm{~g} / \mathrm{L} \text {, } \\
\text { Agitation speed: } 50-350 \mathrm{rpm} \text {, } \\
\text { Temperature: } 290-305 \mathrm{~K} \text {, } \\
\text { Contact time: } 10-100 \mathrm{~min} \text {, } \\
\text { Particle size: } 32.53 \mu \mathrm{m}\end{array}$ & $\mathrm{T}$ & PSO & 725 & [113] \\
\hline $\begin{array}{l}\text { Activated carbons obtained coconut } \\
\text { leaves impregnated with } \mathrm{FeCl}_{3} \\
\text { (carbonized at } 700{ }^{\circ} \mathrm{C} \text { ) }\end{array}$ & $\mathrm{MB}$ & $\begin{array}{l}\text { Initial concentration: } 30-350 \mathrm{mg} / \mathrm{L} \text {, } \\
\text { Initial pH: } 3-11 \text {, } \\
\text { Adsorbent dosage: } 0.2-2.5 \mathrm{~g} / \mathrm{L} \text {, } \\
\text { Rotational speed: } 120 \text { strokes/min, } \\
\text { Temperature: } 303 \mathrm{~K} \text {, } \\
\text { Contact time: } 0-180 \mathrm{~min} \text {, } \\
\text { Particle size: } 150-212 \mu \mathrm{m}\end{array}$ & $\mathrm{L}$ & PSO & 66 & [112] \\
\hline $\begin{array}{l}\text { Coconut leaf } \\
\text { mesoporous-activated carbon } \\
\text { prepared via } \\
\text { microwave-induced } \mathrm{H}_{3} \mathrm{PO}_{4} \\
\text { activation process }\end{array}$ & MB & $\begin{array}{l}\text { Initial concentration: } 50-350 \mathrm{mg} / \mathrm{L} \text {, } \\
\text { Initial pH: } 3-10 \text {, } \\
\text { Adsorbent dosage: } 0.2-10 \mathrm{~g} / \mathrm{L} \text {, } \\
\text { Shaking speed: } 90 \text { strokes } / \mathrm{min} \text {, } \\
\text { Temperature: } 30^{\circ} \mathrm{C} \text {, } \\
\text { Contact time: } 0-360 \text {, } \\
\text { Particle size: } 150-212 \mu \mathrm{m} \text { (fixed) }\end{array}$ & $\mathrm{L}$ & PSO & 250 & [114] \\
\hline $\begin{array}{l}\text { Activated carbon from fallen } \\
\text { coconut leaves prepared by } \\
\text { thermal carbonization using } \\
\text { KOH-activation method }\end{array}$ & $\mathrm{MB}$ & $\begin{array}{l}\text { Initial concentration: } 30-400 \mathrm{mg} / \mathrm{L} \text {, } \\
\text { Initial pH: } 3-11 \text {, } \\
\text { Adsorbent dosage: } 1 \mathrm{~g} / \mathrm{L} \text {, } \\
\text { Shaking speed: } 120 \text { strokes/min, } \\
\text { Temperature: } 303-323 \mathrm{~K} \text {, } \\
\text { Contact time: } 5-300 \mathrm{~min} \text {, } \\
\text { Particle size: } 150-212 \mu \mathrm{m} \text { (fixed) }\end{array}$ & $\mathrm{L}$ & PSO & 147.1 & [115] \\
\hline $\begin{array}{l}\text { Activated carbons obtained guava } \\
\text { leaf (impregnated with } \mathrm{H}_{3} \mathrm{PO}_{4} \\
\text { (carbonized at } 300{ }^{\circ} \mathrm{C} \text { ) }\end{array}$ & $\mathrm{CR}$ & $\begin{array}{l}\text { Initial concentration: } 10-50 \mathrm{mg} / \mathrm{L} \text {, } \\
\text { Initial } \mathrm{pH} \text { : } 3 \text {, } \\
\text { Adsorbent dosage: } 0.5 \mathrm{~g} / \mathrm{L} \text {, } \\
\text { Agitation speed: } 120 \mathrm{rpm} \text {, } \\
\text { Temperature: } 303-323 \mathrm{~K} \text {, } \\
\text { Contact time: } 0-160 \mathrm{~min} \text {, } \\
\text { Particle size: } \leq 106 \mu \mathrm{m}\end{array}$ & $\mathrm{F}$ & PSO & 47.62 & [116] \\
\hline $\begin{array}{l}\text { Activated carbons obtained berry } \\
\text { leaves impregnated with } \mathrm{H}_{3} \mathrm{PO}_{4} \\
\text { (carbonized at } 773 \mathrm{~K} \text { ) }\end{array}$ & EBT & $\begin{array}{l}\text { Initial concentration: } 50-500 \mathrm{mg} / \mathrm{L} \text {, } \\
\text { Initial pH: NA } \\
\text { Adsorbent dosage: } 0.4-3.2 \mathrm{~g} / \mathrm{L} \text {, } \\
\text { Agitation speed: } 140 \mathrm{rpm}, \\
\text { Temperature: } 303-323 \mathrm{~K} \text {, } \\
\text { Contact time: } 0-4 \mathrm{~h}, \\
\text { Particle size: NA }\end{array}$ & $\mathrm{L}$ & PSO & 133.33 & [118] \\
\hline
\end{tabular}

${ }^{\mathrm{a}}$ Key: $\mathrm{L}=$ Langmuir, $\mathrm{F}=$ Freundlich, $\mathrm{T}=$ Temkin, $\mathrm{PFO}=$ Pseudo-first order, $\mathrm{PSO}=$ Pseudo-second order, NA = Not available.

into "carbon burn off" leading to a well-developed pore on GLAC samples. Similarly, the CR-dye adsorption onto GLAC was analysed by four different isotherm models: Freundlich, Langmuir, DubininRadushkevich (D-R) and Temkin isotherms. Freundlich isotherm was found to fit the adsorption data most. Adsorption rate constants were evaluated by PFO, PSO Elovich and intraparticle diffusion (IPD) models. It was evident that CR-dye adsorption onto GLAC was best described by PSO-kinetic model. Critical observation revealed the involvement of IPD in process of adsorption and the mean sorption energy obtained from $\mathrm{D}$ $\mathrm{R}$ isotherm established that physical sorption was involved. Thermodynamically, CR dye adsorption onto GLAC was spontaneous and exothermic in nature. The maximum adsorption was $47.62 \mathrm{mg} / \mathrm{g}$, thus their study revealed the feasibility of GLAC as effective adsorbent for CR dye removal from aqueous solution.

Bello et al. [41] prepared activated carbon from novel and new adsorbent by impregnation method. Moringa oleifera leaf (MOL) was 
impregnated with $\mathrm{NaOH}$ and $\mathrm{H}_{2} \mathrm{SO}_{4}$ respectively. Though, the dye adsorption study was not reported, authors asserted that the influence of both acid and base activations on the surface chemistry of Moringa oleifera leaf were significant. Both $\mathrm{NaOH}$ and $\mathrm{H}_{2} \mathrm{SO}_{4}$ activation of MOL sample decreased the ash contents and left numbers of tiny pores. Concurrently, $\mathrm{H}_{2} \mathrm{SO}_{4}$ and $\mathrm{NaOH}$ activations decomposed the carbon precursors leading to creation of some fresh/new pores and voids [41]. The $\mathrm{NaOH}$ and $\mathrm{H}_{2} \mathrm{SO}_{4}$ activation used permeated such tiny pores or voids, thereby increasing the surface area of contact and interaction of the activating agents with carbon precursors. This greatly, promoted the released of volatile matter from the carbon structures and widened the micropores in the original carbon structures converting them into mesopores. Among others, the proximate analysis revealed higher fixed carbon contents $(69.14 \pm 0.01)$ with $\mathrm{H}_{2} \mathrm{SO}_{4}$-activated MOL. It was concluded that $\mathrm{H}_{2} \mathrm{SO}_{4}$ activated carbon was a promising material for pollutants (e.g. dyes) remediation.

In addition, activated carbon prepared from spent black tea leaves via microwave-assisted method (MASTL) were investigated for Eriochrome Black T (EBT) sequestration from aqueous solution [117]. $\mathrm{A} \mathrm{pH}_{\mathrm{pzc}}$ of 4.6 was obtained for MASTL. The influence of operating conditions including exposure time, $\mathrm{pH}$, solution temperature, adsorbent dosage and EBT-concentrations were studied in order to estimate the interactions between EBT and MASTL. Freundlich, Langmuir, D-R and Temkin models were utilized to test the adsorption data. Langmuir isotherm fitted most with $242.72 \mathrm{mg} / \mathrm{g}$ monolayer adsorption capacity at $25{ }^{\circ} \mathrm{C}$. Also, kinetic study agreed well with PSO-model. Thermodynamically, adsorption isotherm was largely dependent on temperatures. The mechanism of adsorption process followed physisorption occurring spontaneously with absorption of heat. This investigation therefore confirmed the possibility of using MASTL in cleaning-up polluted environments and as low-cost and new adsorbent that is effective for EBT removal from aqueous solutions.

The use of activated carbons obtained from berry leaves impregnated with $\mathrm{H}_{3} \mathrm{PO}_{4}$, (HMBL) for remediation of Eriochrome Black T (EBT) have been investigated [118]. Their study revealed that HMBL has high carbon content with high surface area of $825 \mathrm{~m}^{2} / \mathrm{g}$ with average pore diameter of $3.31 \mathrm{~nm}$. It was also observed that due to C$\mathrm{H}_{3} \mathrm{PO}_{4}$ reactions, there was also an increased adsorbent porosity as well as creation of new pores owing to loss of volatile contents in forms of $\mathrm{CO}_{2}$ and $\mathrm{CO}$. At low concentration of $50 \mathrm{mg} / \mathrm{L}$, EBT removal gave a maximum percentage approximately $96 \%$ whereas at high concentration (500 mg/L) approximately 60\% removal was obtained under the same conditions. Moreover, under this condition at $C_{0}=$ $200 \mathrm{mg} / \mathrm{L}$, the optimum EBT percentage removal was approx. 92\%. Equilibrium was attained within $1 \mathrm{~h}$ and after $1.5 \mathrm{~h}$; it roughly became asymptotic. With an increase in temperature, EBT adsorption decreases. Kinetic and isotherm models were best described by PSO and Langmuir respectively with adsorption capacity of $133.33 \mathrm{mg} / \mathrm{g}$. However, the adsorption thermodynamics of EBT dye adsorption onto HMBL was exothermic and spontaneous in nature. Regeneration studies using $0.1 \mathrm{M}$ $\mathrm{NaOH}$ shown confirmed the reusability of the adsorbent without loss of adsorption capacity. The estimated cost of HMBL was USD 10.684 per $\mathrm{kg}$, it is cost effective when compared to CAC which costs USD 172.96 per $\mathrm{kg}$. Their study revealed that HMBL was a good low cost promising alternative for EBT removal than CAC.

In summary, Table 3 presents the list of various carbonaceous adsorbents derived from leaves with their isotherms, kinetics and corresponding maximum monolayer adsorption capacities.

\subsection{Modified leaf-derived adsorbents}

Durian leaf powder was modified with $\mathrm{NaOH}$ to incorporate $-\mathrm{COO}^{-}$ groups on the Durian leaf powder for improved performance in adsorbing MB dye [119]. The effects of $\mathrm{NaOH}$ on durian leaf powder (DLP) was used to examine the interaction between the NaOH-DLP adsorbent and MB dye using different characterization techniques. Batch adsorption studies were employed to investigate various parameters governing the MB-dye adsorption process. Langmuir model $\left(\mathrm{R}^{2}>\right.$ 0.989 ) best described the equilibrium data with $125 \mathrm{mg} / \mathrm{g}$ maximum monolayer adsorption capacity. Adsorption kinetic correlated well with PSO-model indicating that adsorption process of MB dye onto $\mathrm{NaOH}-\mathrm{DLP}$ followed chemisorption mechanism. It was then asserted that $\mathrm{NaOH}-\mathrm{DLP}$ was a good candidate for removing MB from wastewater. Similarly, Gong et al. [120] investigated the abatement of MB dye from aqueous solution by employing modified dead leaves of plane trees with $\mathrm{NaOH}$. The effects of different variables was studied and the results showed that at $100 \mathrm{mg} / \mathrm{L}$ the initial dye concentration, $2.5 \mathrm{~g} / \mathrm{L}$ biosorbent at $\mathrm{pH}$ of 7 and ambient temperature was the preferred adsorption condition for abating MB dyes from aqueous solution. The removal of $\mathrm{MB}$ using $\mathrm{NaOH}$-modified-dead leaves resulted in maximum removal capacity of $203.28 \mathrm{mg} / \mathrm{g}$ depicting higher affinity for the removal of MB dye than the unmodified-dead leaves with removal capability of $145.62 \mathrm{mg} / \mathrm{g}$. Kinetic and isotherm of sorption was best described by Langmuir- and PSO-models.

Likewise, removal of Acid blue 113 (AB113) dye was investigated using Prunus dulcis (almond) fallen leaves with $\mathrm{NaOH}$ and surfactant treatments using batch adsorption method [60]. The optimum condition for maximum AB113-dye removal was attained at the adsorbent dosage of $10 \mathrm{~g} / \mathrm{L}$ for $\mathrm{NaOH}$ treated Prunus dulcis fallen leaves and $3 \mathrm{~g} / \mathrm{L}$ for surfactant modified-Prunus dulcis fallen leaves at $2.5 \mathrm{~h}$ contact time and temperature of $293 \mathrm{~K}$. By comparison, surfactant modified-Prunus dulcis showed better removal performance (approximately 100\%) than the $\mathrm{NaOH}$ activated Prunus dulcis fallen leaves. The PSO model best described the kinetics of adsorption while Temkin and Langmuir isotherm models best fitted the equilibrium data. The adsorption was spontaneous and exothermic in nature. Under optimization condition, the maximum adsorption capacity for surfactant-modified (i.e. $97.09 \mathrm{mg} / \mathrm{g}$ ) Prunus dulcis fallen leaves was almost four times the one obtained for $\mathrm{NaOH}$ treated biosorbent $(25.51 \mathrm{mg} / \mathrm{g})$. Regeneration studies with other applications demonstrated potential of the Prunus dulcis fallen leaves for AB25 dye sequestration for several cycles. On the other hands, Jain and Gogate prepared new sorbents from NaOH-modifiedFicus racemosa leaves for sequestration of acid blue 25 (AB25) [121]. Batch adsorption experiment was employed to determine the influence of operational parameters on the extent of AB25 dye removal. They established the optimum AB25 dye removal to be $\mathrm{pH}$ of 2, the sorbent dosage of $4 \mathrm{~g} / \mathrm{L}, 3 \mathrm{~h}$ for optimum contact time and $323 \mathrm{~K}$ as optimum temperature. Kinetic study was best described by PSO-model and both Langmuir and Temkin isotherms best described the adsorption isotherms. Thermodynamic investigations confirmed the spontaneity and endothermic in nature of the adsorption process. Adsorption capacity of AB25 dye onto $\mathrm{NaOH}$-modified-Ficus racemosa leaves was determined to be $83.33 \mathrm{mg} / \mathrm{g}$. Finally, desorption-adsorption studies for AB25 dye onto $\mathrm{NaOH}$-modified-Ficus racemosa leaves were successfully conducted for seven cycles indicating that the adsorbent can be reused for several times for dye removal.

Citric acid-modified Ricinus communis leaves (CALRC) were also studied for sequestering MB dye from aquatic effluents [122]. Batch adsorption experiments were used to investigate the influence of operational parameters. The maximum MB dye uptake observed at $\mathrm{pH}$ of 5.26 for CALRC was $81.29 \%$. Langmuir model fitted best, kinetic studies showed a good agreement with PSO-model and IPD. From the experimental results, the authors concluded that citric acid-modified leaves of Ricinus communis leaves is expected to be an inexpensive candidate for water and wastewater remediation purposes.

Goswami and Phukan enhanced the sorption capacities of four dyes: methylene blue (MB), rhodamine B (RhB), brilliant green (BG), orange green $(\mathrm{OG})$ and crystal violet $(\mathrm{CV})$ dyes using both raw and sulfonic acid modified activated carbon prepared from matured tea leaf adsorbent [123]. After modification, the sulfonic acid modified matured tea leaf activated carbon revealed that adsorption capacity for $\mathrm{MB}, \mathrm{RhB}$, BG and CV dyes were enhanced whereas the opposite result was 
Table 4

Modified leaf-derived adsorbents used for dyes removal.

\begin{tabular}{|c|c|c|c|c|c|c|}
\hline Adsorbents & Dye & Initial conditions & $\begin{array}{l}\text { Isotherm } \\
\text { models }\end{array}$ & $\begin{array}{l}\text { Kinetic } \\
\text { models }\end{array}$ & $\begin{array}{l}\text { Maximum adsorption } \\
\text { capacity }(\mathrm{mg} / \mathrm{g})\end{array}$ & Refs. \\
\hline Modified durian leaf with $\mathrm{NaOH}$ & $\mathrm{MB}$ & $\begin{array}{l}\text { Initial concentration: } 10-40 \mathrm{mg} / \mathrm{L} \text {, } \\
\text { Initial pH: } 2-10 \text {, } \\
\text { Adsorbent dosage: } 0.4-2 \mathrm{~g} / \mathrm{L} \text {, } \\
\text { Agitation speed: } 120 \mathrm{rpm} \text {, } \\
\text { Temperature: } \mathrm{RT} \text {, } \\
\text { Contact time: } 0-180 \mathrm{~min} \text {, } \\
\text { Particle size: } 212 \mu \mathrm{m}\end{array}$ & $\mathrm{L}$ & PSO & 125 & [119] \\
\hline $\begin{array}{l}\text { Modified dead leaves of plane trees with } \\
\mathrm{NaOH}\end{array}$ & MB & $\begin{array}{l}\text { Initial concentration: } 100-500 \mathrm{mg} / \mathrm{L} \text {, } \\
\text { Initial pH: } 3-13 \text {, Adsorbent dosage: } 2.5-10.0 \mathrm{~g} / \mathrm{L} \text {, } \\
\text { Temperature: } 293.15 \mathrm{~K} \text {, } \\
\text { Contact time: } 0-500 \mathrm{~min} \text {, } \\
\text { Particle size: } 0.45-0.9 \mathrm{~mm}\end{array}$ & $\mathrm{~L}$ & PSO & 243.902 & [120] \\
\hline $\begin{array}{l}\text { Modified Prunus dulcis (almond) fallen } \\
\text { leaves with surfactant }\left(\mathrm{C}_{19} \mathrm{H}_{42} \mathrm{BrN}\right)\end{array}$ & AB113 & $\begin{array}{l}\text { Initial concentration: } 50-450 \mathrm{mg} / \mathrm{L} \text {, } \\
\text { Initial pH: Natural pH of the dye, Adsorbent dosage: } 0.2-6 \mathrm{~g} / \mathrm{L} \text {, } \\
\text { Agitation speed: } 150 \mathrm{rpm} \text {, } \\
\text { Temperature: } 293-323 \mathrm{~K} \text {, } \\
\text { Contact time: } 0-5 \mathrm{~h} \text {, } \\
\text { Particle size: } 53-106 \mu \mathrm{m} \text {, } \\
\text { Ionic strength: } \mathrm{NaCl} 0.01 \text { to } 0.1 \mathrm{~mol} / \mathrm{L}\end{array}$ & $\mathrm{L}, \mathrm{T}$ & PSO & 97.09 & {$[60]$} \\
\hline Modified Ficus racemosa with $\mathrm{NaOH}$ & AB25 & $\begin{array}{l}\text { Initial concentration: } 50-400 \mathrm{mg} / \mathrm{L} \mathrm{mg} / \mathrm{L} \text {, } \\
\text { Initial } \mathrm{pH}: 2-10 \text {, } \\
\text { Adsorbent dosage: } 1-10 \mathrm{~g} / \mathrm{L} \text {, } \\
\text { Agitation speed: } 150 \mathrm{rpm} \text {, } \\
\text { Temperature: } 293-323 \mathrm{~K}, \\
\text { Contact time: } 0-5 \mathrm{~h}\end{array}$ & $\mathrm{~L}, \mathrm{~T}$ & PSO & 83.33 & [121] \\
\hline $\begin{array}{l}\text { Sulfonic acid modified activated carbon } \\
\text { prepared from matured tea leaf }\end{array}$ & RhB & $\begin{array}{l}\text { Initial concentration: } 200-800 \mathrm{mg} / \mathrm{L} \text {, } \\
\text { Initial pH: } 2-11 \text {, } \\
\text { Adsorbent dosage: } 0.4-3 \mathrm{~g} / \mathrm{L} \text {, } \\
\text { Agitation speed: } 200 \mathrm{rpm} \text {, } \\
\text { Temperature: } 303-323 \mathrm{~K} \text {, } \\
\text { Contact time: } 0 \text { to } \approx 275 \mathrm{~min} \text {, } \\
\text { Ionic strength: } \mathrm{NaCl} 0-0.5 \mathrm{~mol} / \mathrm{L}\end{array}$ & $\mathrm{L}$ & PSO & 757.6 & [123] \\
\hline $\begin{array}{l}\text { Sulfonic acid modified activated carbon } \\
\text { prepared from matured tea leaf }\end{array}$ & MB & $\begin{array}{l}\text { Initial concentration: NA, } \\
\text { Initial pH: NA, } \\
\text { Adsorbent dosage: } 0.4 \mathrm{~g} / \mathrm{L} \text {, } \\
\text { Agitation speed: } 200 \mathrm{rpm} \text {, } \\
\text { Temperature: } 303 \mathrm{~K} \text {, } \\
\text { Contact time: NA, }\end{array}$ & $\mathrm{L}$ & - & 714.3 & [123] \\
\hline $\begin{array}{l}\text { Sulfonic acid modified activated carbon } \\
\text { prepared from matured tea leaf }\end{array}$ & BG & $\begin{array}{l}\text { Initial concentration: } \mathrm{NA} \text {, } \\
\text { Initial pH: NA, } \\
\text { Adsorbent dosage: } 0.4 \mathrm{~g} / \mathrm{L} \text {, } \\
\text { Agitation speed: } 200 \mathrm{rpm} \text {, } \\
\text { Temperature: } 303 \mathrm{~K} \text {, } \\
\text { Contact time: NA, }\end{array}$ & $\mathrm{L}$ & - & 704.2 & [123] \\
\hline $\begin{array}{l}\text { Sulfonic acid modified activated carbon } \\
\text { prepared from matured tea leaf }\end{array}$ & $\mathrm{CV}$ & $\begin{array}{l}\text { Initial concentration: NA, } \\
\text { Initial pH: NA, } \\
\text { Adsorbent dosage: } 0.4 \mathrm{~g} / \mathrm{L} \text {, } \\
\text { Agitation speed: } 200 \mathrm{rpm} \text {, } \\
\text { Temperature: } 303 \mathrm{~K} \text {, } \\
\text { Contact time: NA, }\end{array}$ & $\mathrm{L}$ & - & 653.5 & [123] \\
\hline $\begin{array}{l}\text { Sulfonic acid modified activated carbon } \\
\text { prepared from matured tea leaf }\end{array}$ & OG & $\begin{array}{l}\text { Initial concentration: } 200-500 \mathrm{mg} / \mathrm{L} \text {, } \\
\text { Initial pH: } 2-11 \text {, } \\
\text { Adsorbent dosage:: } 0.5-3.5 \mathrm{~g} / \mathrm{L} \text {, } \\
\text { Agitation speed: } 200 \mathrm{rpm} \text {, } \\
\text { Temperature: } 303-323 \mathrm{~K} \text {, } \\
\text { Contact time: } 0 \text { to } \approx 275 \mathrm{~min} \text {, } \\
\text { Ionic strength: } \mathrm{NaCl} 0-0.5 \mathrm{~mol} / \mathrm{L}\end{array}$ & $\mathrm{L}$ & PSO & 105.7 & [123] \\
\hline $\begin{array}{l}\text { Modified pineapple leaf powder with } \\
\text { surfactant HDTMA-Br }\end{array}$ & MB & $\begin{array}{l}\text { Initial concentration: } 5-1000 \mathrm{mg} / \mathrm{L} \text {, } \\
\text { Initial pH: } 3-11 \text {, } \\
\text { Adsorbent dosage: } 10 \mathrm{~g} / \mathrm{L} \text {, } \\
\text { Agitation speed: } 250 \mathrm{rpm} \text {, } \\
\text { Temperature: } 308.15-333.15 \mathrm{~K} \text {, } \\
\text { Contact time: } 2 \mathrm{~h} \text {, } \\
\text { Particle size: }<425 \mu \mathrm{m}\end{array}$ & $\mathrm{L}$ & - & 52.6 & [124] \\
\hline $\begin{array}{l}\text { Modified pineapple leaf powder with } \\
\text { surfactant HDTMA-Br }\end{array}$ & MO & $\begin{array}{l}\text { Initial concentration: } 5-1000 \mathrm{mg} / \mathrm{L} \text {, } \\
\text { Initial pH: } 3-11 \text {, } \\
\text { Adsorbent dosage: } 10 \mathrm{~g} / \mathrm{L} \text {, } \\
\text { Agitation speed: } 250 \mathrm{rpm} \text {, } \\
\text { Temperature: } 308.15-333.15 \mathrm{~K}, \\
\text { Contact time: } 2 \mathrm{~h} \text {, } \\
\text { Particle size: }<425 \mu \mathrm{m}\end{array}$ & $\mathrm{L}$ & - & 47.6 & [124] \\
\hline $\begin{array}{l}\text { Modified Calotropis procera leaf powder with } \\
\mathrm{HCl}\end{array}$ & $\mathrm{MB}$ & $\begin{array}{l}\text { Initial concentration: } 200-1600 \mathrm{mg} / \mathrm{L} \text {, } \\
\text { Initial } \mathrm{pH} \text { : } 2-12 \text {, } \\
\text { Adsorbent dosage: } 16-160 \mathrm{~g} / \mathrm{L} \text {, }\end{array}$ & $\mathrm{L}$ & - & 192.31 & [125] \\
\hline
\end{tabular}


Table 4 (continued)

\begin{tabular}{|c|c|c|c|c|c|c|}
\hline Adsorbents & Dye & Initial conditions & $\begin{array}{l}\text { Isotherm } \\
\text { models }\end{array}$ & $\begin{array}{l}\text { Kinetic } \\
\text { models }\end{array}$ & $\begin{array}{l}\text { Maximum adsorption } \\
\text { capacity }(\mathrm{mg} / \mathrm{g})\end{array}$ & Refs. \\
\hline & & $\begin{array}{l}\text { Agitation speed: } 120 \mathrm{rpm} \text {, } \\
\text { Temperature: } 306 \mathrm{~K} \text { and } 323 \mathrm{~K} \text {, } \\
\text { Contact time: } 10-100 \mathrm{~min} \text {, } \\
\text { Particle size: }<150 \mu \mathrm{m}\end{array}$ & & & & \\
\hline $\begin{array}{l}\text { Modified C. camphora leaves powder with } \\
\text { NaOH }\end{array}$ & MV & $\begin{array}{l}\text { Initial concentration: } 10-90 \mathrm{mg} / \mathrm{L} \text {, } \\
\text { Initial pH: } 2-10 \text {, } \\
\text { Adsorbent dosage: } 0.2,-0.6 \mathrm{~g} / \mathrm{L} \text {, } \\
\text { Agitation speed: } 120 \text { and } 130 \mathrm{rpm} \text {, } \\
\text { Temperature: } 283.5-323.5 \text {, } \\
\text { Contact time: } 0 \text { to } \approx 700 \mathrm{~min} \text {, } \\
\text { Particle size: } 100-300 \mathrm{~mm}\end{array}$ & $\mathrm{~L}$ & PSO & 206.6 & [126] \\
\hline $\begin{array}{l}\text { Modified Glossogyne tenuifolia with } \mathrm{NH}_{3} \text { and } \\
\qquad \mathrm{C}_{12} \mathrm{H}_{24} \mathrm{O}_{2}\end{array}$ & CR & $\begin{array}{l}\text { Initial concentration: } 100-500 \mathrm{mg} / \mathrm{L} \text {, } \\
\text { Initial pH: } 2-10 \text {, } \\
\text { Adsorbent dosage: } 12 \mathrm{~g} / \mathrm{L} \text {, } \\
\text { Agitation speed: } 100 \mathrm{rpm} \text {, } \\
\text { Temperature: } 293-323 \mathrm{~K}, \\
\text { Contact time: } 0-24 \mathrm{~h}, \\
\text { Average particle size: } 0.4 \mathrm{~mm}\end{array}$ & $\mathrm{~F}$ & PSO & 37.18 & [127] \\
\hline $\begin{array}{l}\text { Modified Glossogyne tenuifolia with } \mathrm{NH}_{3} \text { and } \\
\mathrm{C}_{12} \mathrm{H}_{24} \mathrm{O}_{2}\end{array}$ & (1) & $\begin{array}{l}\text { Initial concentration: } 100-500 \mathrm{mg} / \mathrm{L} \text {, } \\
\text { Initial pH: } 2-10 \text {, } \\
\text { Adsorbent dosage: } 2 \mathrm{~g} / \mathrm{L} \text {, } \\
\text { Agitation speed: } 100 \mathrm{rpm} \text {, } \\
\text { Temperature: } 293-323 \mathrm{~K} \text {, } \\
\text { Contact time: } 0-24 \mathrm{~h}, \\
\text { Average particle size: } 0.4 \mathrm{~mm}\end{array}$ & $\mathrm{~F}$ & PSO & 370.4 & [127] \\
\hline Ficus racemosa leaves activated with $\mathrm{NaOH}$ & AV17 & $\begin{array}{l}\text { Initial concentration: } \mathrm{NA} \text {, } \\
\text { Initial pH: } 2-10 \text {, } \\
\text { Adsorbent dosage: } 0.5-5 \mathrm{~g} / \mathrm{L} \text {, } \\
\text { Agitation speed: } 150 \mathrm{rpm} \text {, } \\
\text { Temperature: } 293-323 \mathrm{~K} \text {, } \\
\text { Contact time: } 0-360 \mathrm{~min}, \\
\text { Particle size: } 53-106 \mu \mathrm{m}\end{array}$ & $\mathrm{L}$ & PSO & 119.05 & [88] \\
\hline
\end{tabular}

${ }^{\mathrm{a}}$ Key: $\mathrm{L}=$ Langmuir, $\mathrm{F}=$ Freundlich, $\mathrm{T}=$ Temkin, PFO = Pseudo-first order, PSO = Pseudo-second order, RT = Room temperature, NA: Not available.

observed for OG dye. Process variables significantly affected the dye adsorption onto sulfonic acid modified matured tea leaf activated carbon adsorbent thereby revealing that adsorption was favourable when sulfonic acid modified matured tea leaf activated carbon was used for dye uptake (MB, RhB, BG and CV) under study. As for RhB and OG dyes, the process of adsorption showed a significant dye uptake during the first hour thereafter it was gradual until the equilibrium was attained. Generally, small increased in adsorption capacity was observed with the increase of ionic strength from 0 to $0.5 \mathrm{~mol} \mathrm{NaCl} / \mathrm{L}$. Langmuir model best described the equilibrium adsorption data. The study also showed that adsorption of RhB dye onto sulfonic acid modified matured tea leaf was found to be more favourable than other dyes under study with highest removal capacity of $757.6 \mathrm{mg} / \mathrm{g}$ for RhB dye. The kinetic of adsorption is most fitted with PSO-model. Thermodynamics studies established the endothermic and spontaneous nature of the adsorption process.

Moreover, the surfactant-modified-pineapple leaf powder (SMPLP) was investigated for its variability to remove $\mathrm{MB}$ and methyl orange (MO) dyes from aqueous solutions [124]. Different concentrations of PLP (pineapple leaf powder) were allowed to interact with cationic surfactant, hexadecyltrimethylammonium bromide (HDTMA-Br) to form SMPLP. Batch adsorption technique was employed to study the effects of different parameters toward MB and MO dye adsorption. Langmuir adsorption isotherm model was found to give the best fit for both dyes. The maximum adsorption capacity of MB $(52.6 \mathrm{mg} / \mathrm{g})$ and MO (47.6 mg/g) was achieved using PLP and PLP treated with $4.0 \mathrm{mM}$ HDTMA-Br, respectively. In the case of MO, adsorption of MO drastically increased when SMPLP was 4.0 mM of HDTMA-Br than when HDTMABr was 1.0 and $2.5 \mathrm{mM}$ respectively showing that HDTMA-Br concentrations have profound influence on the interaction of SMPLP and MO. The author generally concluded that SMPLP is a promising adsorbent for freeing the aqueous solution from both $\mathrm{MO}$ and $\mathrm{MB}$ dyes.
Oyelude and Owusu reported the possibility of $\mathrm{HCl}$-modified Calotropis procera leaf powder (HCl-ACLP) as efficient adsorbent for abating MB dye from aqueous solutions [125]. The effects of process variables were observed and it was noted that MB dye removal was affected by agitation time and initial dye concentrations. The optimum contact time was $80 \mathrm{~min}$ and the amount adsorbed decreased from $1.1 \mathrm{mg} / \mathrm{g}$ to $0.1 \mathrm{mg} / \mathrm{g}$ as HCl-ACLP dosage increases from $16 \mathrm{~g} / \mathrm{L}$ to $160 \mathrm{~g} / \mathrm{L}$. The percentage removal of MB dye increases from $87 \%$ to $95 \%$ and optimum MB dye uptake attained was between $\mathrm{pH} 8$ and 12. Langmuir isotherm fitted most. Adsorption process was reported to be feasible and endothermic having $192.31 \mathrm{mg} / \mathrm{g}$ maximum monolayer adsorption capacity with $\Delta G^{0}$ value of- $17.23 \mathrm{~kJ} / \mathrm{mol}$.

Tanga et al. reported an enhanced sorption of cationic dye, methyl violet (MV) dye, from aqueous solution with $\mathrm{NaOH}$-modified $C$. camphora leaves powder (N-CLP), compared with $C$. camphora leaves powder (CLP) [126]. The experimental results showed that the pH exerts a strong influence on the adsorption of MV dye onto N-CLP. The adsorption process was conducted at $\mathrm{pH}$ ranging from 4.0-10.0. Electrostatic attraction and $\pi-\pi$ transition could be involved in adsorption. The adsorption process for MV dye on N-CLP followed PSO-kinetics model and the equilibrium adsorption data fitted well to Langmuir isotherm. The negative values of free energy change indicated the spontaneous nature of the adsorption and the positive values of enthalpy change suggested the endothermic nature of the adsorption process. After three adsorption/desorption cycles, the uptake capacity of N-CLP was found to decrease by $15 \%$, thus indicating that adsorbent had good reuse performance.

Besides, malachite green (MG) and Congo red (CR) dyes were successfully removed from the aqueous solutions usingmodifiedGlossogyne tenuifolia leaves (MGTL) using aqueous ammonia $\left(\mathrm{NH}_{3 \mathrm{aq}}\right)$, lauric acid $\left(\mathrm{C}_{12} \mathrm{H}_{24} \mathrm{O}_{2}\right)$ and combination of both as chemical agents [127]. The influences of $\mathrm{NH}_{3(\mathrm{aq})}$ and lauric acid at different 
Table 5

List of thermodynamic parameters for the adsorption of dyes onto leaf-based adsorbents.

\begin{tabular}{|c|c|c|c|c|c|c|}
\hline Adsorbent & Dye & $\mathrm{T}(\mathrm{K})$ & $\Delta G^{\mathrm{o}}(\mathrm{kJ} / \mathrm{mol})$ & $\Delta H^{\mathrm{o}}(\mathrm{kJ} / \mathrm{mol})$ & $\Delta S^{\mathrm{o}}(\mathrm{kJ} / \mathrm{mol} \mathrm{K})$ & References \\
\hline \multirow[t]{4}{*}{ Surfactant modified fallen leaves of Prunus Dulcis } & AB113 & 293 & -29.383 & -31.34 & -0.007 & {$[60]$} \\
\hline & & 303 & -29.194 & & & \\
\hline & & 313 & -29.181 & & & \\
\hline & & 323 & -29.167 & & & \\
\hline \multirow[t]{4}{*}{$\mathrm{NaOH}$-treated Ficus racemosa dead leaves ${ }^{\mathrm{a}}$} & AB25 & 293 & -26.62 & 29.85 & 0.192 & {$[121]$} \\
\hline & & 303 & -28.34 & & & \\
\hline & & 313 & -30.31 & & & \\
\hline & & 323 & -32.4 & & & \\
\hline \multirow[t]{4}{*}{$\mathrm{NaOH}$ activated Prunus Dulcis ${ }^{\mathrm{a}}$} & AG25 & 293 & -34.676 & 6.991 & 0.142 & {$[140]$} \\
\hline & & 303 & -36.013 & & & \\
\hline & & 313 & -37.384 & & & \\
\hline & & 323 & -38.965 & & & \\
\hline \multirow[t]{4}{*}{ Leaf powder of Paulownia tomentosa Steud. } & A052 & 298 & 6.42 & -5.63 & -0.04 & \\
\hline & & & & & & [99] \\
\hline & & 308 & 6.81 & & & \\
\hline & & 318 & 7.23 & & & \\
\hline \multirow{4}{*}{$\mathrm{NaOH}$ activated Ficus racemosa fallen leaves ${ }^{\mathrm{a}}$} & AV17 & 293 & -26.612 & 10.98 & 0.13 & [88] \\
\hline & & 303 & -27.799 & & & \\
\hline & & 313 & -29.076 & & & \\
\hline & & 323 & -30.466 & & & \\
\hline \multirow[t]{4}{*}{ Neem leaf powder (Azadirachta indica) ${ }^{\mathrm{b}}$} & BG & 300 & -7.15 & 5.66 & 0.043 & {$[103]$} \\
\hline & & 303 & -7.28 & & & \\
\hline & & 313 & -7.70 & & & \\
\hline & & 323 & -8.13 & & & \\
\hline \multirow[t]{3}{*}{ Ashoka (Saraca asoca) leaf powder } & BG & 303 & -7.984 & -24.659 & -0.055 & [87] \\
\hline & & 313 & -7.434 & & & \\
\hline & & 323 & -6.884 & & & \\
\hline \multirow[t]{3}{*}{ Pine tree leaves } & BR46 & 298 & -4.71 & 46.44 & 0.17 & {$[101]$} \\
\hline & & 308 & -7.11 & & & \\
\hline & & 318 & -8.13 & & & \\
\hline \multirow[t]{3}{*}{ Princess tree leaf } & BR46 & 298 & -4.29 & -43.76 & -0.13 & {$[102]$} \\
\hline & & 308 & -3.17 & & & \\
\hline & & 318 & -1.64 & & & \\
\hline Glossogyne tenuifolia leaves modified with aqueous ammonia and lauric acid solution & $\mathrm{CR}$ & 293 & -1.474 & 0.004 & 0.006 & \\
\hline & & & & & & {$[127]$} \\
\hline & & 303 & -2.053 & & & \\
\hline & & 313 & -2.142 & & & \\
\hline & & 323 & -2.199 & & & \\
\hline Activated carbons obtained berry leaves impregnated with $\mathrm{H}_{3} \mathrm{PO}_{4}$ & EBT & 303 & -5.937 & -37.149 & -0.103 & {$[118]$} \\
\hline & & 313 & -4.907 & & & \\
\hline & & 323 & -3.877 & & & \\
\hline Pineapple leaf powder & MB & 277 & -24.76 & -5.93 & 0.076 & [95] \\
\hline & & 287 & -25.53 & & & \\
\hline & & 297 & -26.12 & & & \\
\hline & & 307 & -26.83 & & & \\
\hline & & 317 & -27.51 & & & \\
\hline & & 327 & -28.19 & & & \\
\hline Bauhinia purpurea L. leaves powder & MB & $303-323$ & NA & -52.68 & -0.158 & \\
\hline & & & & & & [93] \\
\hline Platanus orientalis leaves powder & $\mathrm{MB}$ & 298 & -0.17 & 9.941 & 0.034 & {$[106]$} \\
\hline & & 313 & -0.70 & & & \\
\hline & & 323 & -0.99 & & & \\
\hline & & 333 & -1.37 & & & \\
\hline Fallen phoenix tree's leaves & MB & 295 & -3.89 & 7.77 & -0.040 & {$[92]$} \\
\hline & & 309 & -4.62 & & & \\
\hline & & 323 & -5.01 & & & \\
\hline Neem leaf powder (Azadirachta indica) ${ }^{\mathrm{C}}$ & MB & 300 & -10.33 & 16.74 & 0.090 & [90] \\
\hline & & 310 & -11.24 & & & \\
\hline & & 320 & -12.14 & & & \\
\hline & & 330 & -13.04 & & & \\
\hline Pineapple leaf powder & $\mathrm{MB}$ & 308.15 & -4.319 & -2.788 & 0.004 & {$[124]$} \\
\hline & & 313.15 & -4.327 & & & \\
\hline & & 333.15 & -4.437 & & & \\
\hline Coconut leaves- $\mathrm{H}_{2} \mathrm{SO}_{4}$ activated carbon & MB & 303 & -9.51 & 29.8 & 0.129 & {$[111]$} \\
\hline & & 313 & -10.2 & & & \\
\hline & & 323 & -12.1 & & & \\
\hline Carbon obtained from leaves of teak (Tectona grandis) and Guava (Psidium guajava) trees & MB & 293 & -21.650 & 23.146 & 0.153 & \\
\hline & & & & & & {$[141]$} \\
\hline & & 303 & -23.324 & & & \\
\hline & & 313 & -24.748 & & & \\
\hline & & 323 & -26.271 & & & \\
\hline Ashoka (Saraca asoca) leaf powder & $\mathrm{MB}$ & 303 & -6.555 & -11.830 & -0.017 & \\
\hline & & 313 & -6.381 & & & [87] \\
\hline
\end{tabular}


Table 5 (continued)

\begin{tabular}{|c|c|c|c|c|c|c|}
\hline Adsorbent & Dye & $\mathrm{T}(\mathrm{K})$ & $\Delta G^{\mathrm{o}}(\mathrm{kJ} / \mathrm{mol})$ & $\Delta H^{\mathrm{o}}(\mathrm{kJ} / \mathrm{mol})$ & $\Delta S^{\mathrm{o}}(\mathrm{kJ} / \mathrm{mol} \mathrm{K})$ & References \\
\hline & & 323 & -6.207 & & & \\
\hline \multirow{3}{*}{ Lotus leaf } & & 293 & -25.67 & 4.7 & 0.103 & [142] \\
\hline & & 303 & -26.57 & & & \\
\hline & & 313 & -27.74 & & & \\
\hline \multirow[t]{5}{*}{ Citric acid treated carbonized bamboo leaves powder } & $\mathrm{MB}$ & 290 & -25.43 & 134.97 & 0.555 & \\
\hline & & & & & & [113] \\
\hline & & 295 & -29.01 & & & \\
\hline & & 300 & -31.83 & & & \\
\hline & & 305 & -33.70 & & & \\
\hline \multirow[t]{3}{*}{ Ashoka (Saraca asoca) leaf powder } & MG & 303 & -4.190 & -6.671 & -0.008 & [87] \\
\hline & & 313 & -4.108 & & & \\
\hline & & 323 & -4.026 & & & \\
\hline \multirow[t]{5}{*}{ Glossogyne tenuifolia leaves modified with aqueous ammonia and lauric acid solution } & MG & 293 & -6.884 & 11.471 & 0.063 & \\
\hline & & & & & & [127] \\
\hline & & 303 & -7.850 & & & \\
\hline & & 313 & -8.120 & & & \\
\hline & & 323 & -9.718 & & & \\
\hline \multirow[t]{3}{*}{ Surfactant-modified pineapple leaf powder } & MO & 308.15 & -3.746 & -8.748 & -0.016 & [124] \\
\hline & & 313.15 & -3.689 & & & \\
\hline & & 333.15 & -3.349 & & & \\
\hline \multirow[t]{3}{*}{ Dead leaves of Posidonia oceanica (L.) ${ }^{\mathrm{d}}$} & MV & 293 & -7.366 & 17.153 & 0.084 & [97] \\
\hline & & 308 & -8.734 & & & \\
\hline & & 318 & -9.646 & & & \\
\hline \multirow{3}{*}{ Activated carbon prepared from matured tea leaf } & OG & 303 & -2.66 & 12.4 & 0.05 & [123] \\
\hline & & 313 & -3.16 & & & \\
\hline & & 323 & -3.65 & & & \\
\hline \multirow[t]{3}{*}{ Sulfonic acid modified activated carbon prepared from matured tea leaf } & OG & 303 & -0.709 & 22.5 & 0.077 & [123] \\
\hline & & 313 & -1.47 & & & \\
\hline & & 323 & -2.24 & & & \\
\hline \multirow[t]{3}{*}{ Activated carbon prepared from matured tea leaf } & RhB & 303 & -3.75 & 50.24 & 0.178 & [123] \\
\hline & & 313 & -5.54 & & & \\
\hline & & 323 & -7.31 & & & \\
\hline \multirow[t]{3}{*}{ Sulfonic acid modified activated carbon prepared from matured tea leaf } & RhB & 303 & -7.32 & 26.8 & 0.113 & [123] \\
\hline & & 313 & -8.45 & & & \\
\hline & & 323 & -9.38 & & & \\
\hline \multirow[t]{3}{*}{ Ashoka (Saraca asoca) leaf powder } & RhB & 303 & -1.783 & -5.941 & -0.014 & [87] \\
\hline & & 313 & -1.646 & & & \\
\hline & & 323 & -1.509 & & & \\
\hline
\end{tabular}

NA: Not available.

a Langmuir: Thermodynamic parameters estimated using $\mathrm{K}_{\mathrm{L}}$ from Langmuir model.

b Thermodynamic parameters estimated at initial dye concentration of $2.07 \times 10^{-2} \mathrm{mmol} / \mathrm{dm}^{3}$.

c Thermodynamic parameters estimated at initial dye concentration of $25 \mathrm{mg} / \mathrm{L}$.

d Thermodynamic parameters estimated at initial dye concentration of $65 \mathrm{mg} / \mathrm{L}$.

concentrations, different liquid to solid ratio, and diverse modification on efficient removal of MG and CR dyes were investigated. Adsorption isotherms and kinetic data fitted well to Freundlich model and PSOmodel. Adsorptions of both CR and MG dye were endothermic and spontaneous in nature. It was therefore confirmed that MGTL leaves have a strong potential to remediate the environment contaminated with MG and CR dyes.

Jain, and Gogate [88] investigated the Ficus racemosa fallen leaves activated with $\mathrm{H}_{2} \mathrm{SO}_{4}$ and $\mathrm{NaOH}$, for adsorption of Acid Violet 17 (AV17) dye. Batch adsorption studies were used to investigate the influence of different adsorption parameters on the extents of AV17 dye adsorption. The removal AV17 dye was higher for $\mathrm{NaOH}$-activated biosorbents under optimized $\mathrm{pH} 2$ and biosorbent dosage $3 \mathrm{~g} / \mathrm{L}$ conditions than $\mathrm{H}_{2} \mathrm{SO}_{4}$-activated biosorbents. Adsorption kinetics favoured PSO-model and isotherm fitted well to Langmuir model. After the modification of raw Ficus racemosa fallen leaves, adsorption capacities was found to increase from 45.25 to 61.35 and 45.25 to $119.05 \mathrm{mg} / \mathrm{g}$ for $\mathrm{H}_{2} \mathrm{SO}_{4}{ }^{-}$ activated and $\mathrm{NaOH}$-activated adsorbents, respectively. In case of $\mathrm{NaOH}$-modified leaves, activation energy $E_{a}$ calculated from Arrhenius equation was estimated to be $7.07 \mathrm{~kJ} / \mathrm{mol}$, indicating a physical adsorption of AV17 dye onto the adsorbent was taken place. Both batch and column experiments also confirmed the suitability of the sorbent for efficient removal of AV17 with the adsorption equilibrium column data best fitted with Thomas model.
In general, Table 4 presented the list of various modified leaf-derived adsorbents with their respective isotherms, kinetics and corresponding maximum monolayer adsorption capacities.

\section{Thermodynamic studies}

On Table 5, are tabulated the thermodynamic parameters (Gibbs free energy $\left(\Delta G^{0}\right)$, enthalpy change $\left(\Delta H^{0}\right)$ and entropy change $\left.\left(\Delta S^{0}\right)\right)$ of the works discussed in this review article. The thermodynamic parameters are very useful because they provide information about the spontaneity and endo- or exo-thermicity of the adsorption process and the increment or decrease of randomness at the solid-liquid interface $[128,129]$. Moreover maybe also used to predict the adsorption mechanisms (e.g. physical and chemical) [85,130,131]. The Eqs. (1) and (2) are used to estimate the $\Delta G^{0}, \Delta H^{0}$ and $\Delta S^{0}$.

$$
\begin{aligned}
& \Delta G^{0}=-R T \ln K \\
& \Delta G^{0}=\Delta H^{0}-\mathrm{T} \Delta S^{0} \\
& \Delta S^{0}=\left(-\frac{\Delta G^{0}}{\mathrm{~T}}\right)-\left(-\frac{\Delta H^{0}}{\mathrm{~T}}\right)
\end{aligned}
$$




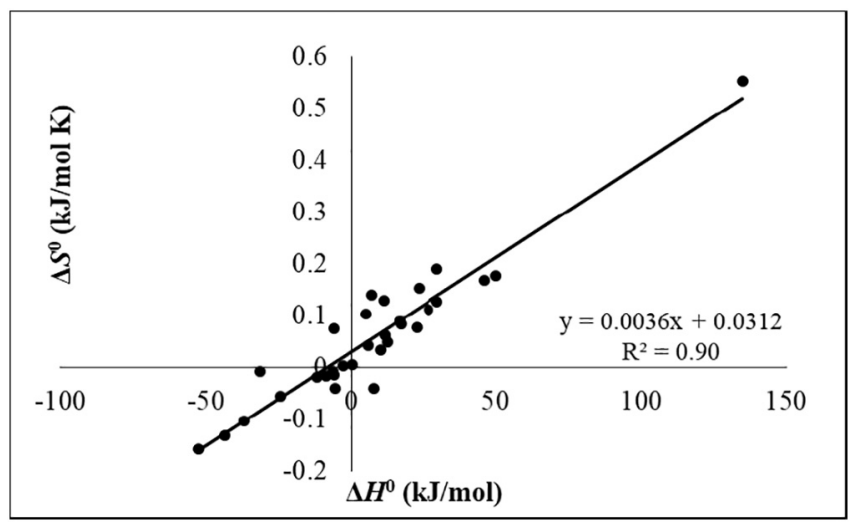

Fig. 3. The enthalpy-entropy compensation plot for the works studied in the present review article.

The constant $R$ is the universal gas constant $(8.314 \mathrm{~J} / \mathrm{mol} \mathrm{K}), T$ is the absolute temperature in Kelvin and $K$ is the equilibrium constant.

Based on Table 5, the thermodynamic parameters estimated in a temperature range $277-327 \mathrm{~K}$ and the adsorption in most cases was found to be spontaneous, endothermic, with increasing in the randomness at the solid-liquid interface. The absolute values of $\Delta G^{0}$ and $\Delta H^{0}$ ranged from 0.17 to 38.965 and from 0.004 to $134.97 \mathrm{~kJ} / \mathrm{mol}$, respectively. Generally, an adsorption process is considered as physical if absolute magnitude $\Delta \mathrm{H}<40 \mathrm{~kJ} / \mathrm{mol}$ and as chemical when $\Delta \mathrm{H}>40 \mathrm{~kJ} / \mathrm{mol}$ [132].

Using the values of $\Delta S^{0}$ and $\Delta H^{0}$ from Table 5 and based on the Eq. (3), a plot of $\Delta S^{0}$ versus $\Delta H^{0}$ reveals a good linear relationship $\left(R^{2}\right.$ $=0.90$ ) (Fig. 3) which indicates the existence of an enthalpy-entropy compensation effect [28,133-137].

The linearity was potentially attributed to an artifact of the assessment method of thermodynamic system parameters since $\Delta S^{0}$ and $\Delta H^{0}$ are not measured independently but they estimated from the linear Van't Hoff plot $[138,139]$.

\section{Conclusions, future perspectives and challenges}

This review has attempted to present a wide range of studies published in the literature so far based on the use of leaf-based adsorbents for the removal of organic dyes. This is because the leaf-based adsorbents are cheap materials, available in large quantities in almost all regions of the world, which required only a few stages of preparation, and in many cases not have another use. The adsorptive removal of organic dyes is a research area in constant development and many efforts have been made by researchers to contribute with a low-cost alternative that can be as efficient as that offered by classical remediation technologies. In the majority of studies, good removal percentages and adsorption capacities were obtained using leaf-based adsorbents. Furthermore, most of these contributions are focused on equilibrium, thermodynamics and kinetic studies of adsorption. However, some future perspectives and challenges have arisen as result of the analysis of this work and are mentioned as follow.

i). As leaves are adsorbents widely available in the nature, it is necessary to obtain new biodegradable, low-cost and efficient leafbased adsorbents for dye removal.

ii). The majority of studies based on leaf-based adsorbents are generally developed in synthetic aqueous solutions, which contain the dye under study only. This lack of application in real samples can limited the potential of the adsorption process, due to the matrix effect caused by the concomitants present in real samples can interfere in the sorption. Thus, future works will include a detailed evaluation of the effect of potential interferences in the sorption and applications in real samples are necessary. iii). The studies discussed in this review article have been mainly made in batch systems, which could be impracticable for industrial applications. Thus, additional works that include leaf-based adsorption studies in continuous systems are very welcome.

iv). Most publications using leaf-based adsorbents are focused on lab-scale. It is essential to work in the scale-up of the adsorption process, not only considering technical aspects but also making an economic analysis to know the overall cost of the process.

$\mathrm{v})$. The majority of leaf-based adsorption process makes the phase separation by centrifugation or filtration. Nevertheless, these steps can be expensive at industrial scales. In this sense, studies that offer alternatives for the separation stage are desirable.

vi). Although numerous mathematical models have been used for single dye adsorption processes, it is necessary to develop new and improved mathematical models that describe matrix systems containing several dyes.

\section{Acknowledgements}

Dr. Laura Bulgariu thanks the support of the Romanian National Authority for Scientific Research, CNCS - UEFISCDI, project number PN-IIIP4-ID-PCE-2016-0500.

Dr. Leticia Escudero thanks the support of Consejo Nacional de Investigaciones Científicas y Técnicas (CONICET), Agencia Nacional de Promoción Científica y Tecnológica (FONCYT) (PICT-BID) and Universidad Nacional de Cuyo (Argentina).

Prof. Olugbenga Solomon Bello and Mr. Kayode Adesina Adegoke acknowledge that their research work obtained supports from Third World Academy of Sciences (TWAS) in form of Research grants; Research Grant number: 11-249 RG/CHE/AF/AC_1_UNESCO FR: 3240262674 (2012), 15-181 RG/CHE/AF/AC_1_: 3240287083 (2015), NRF-TWAS Doctoral Fellowship (UID: 105453 \& Reference: SFH160618172220) and 2016 TETFund Institution Based Research Intervention respectively.

Prof. Michael Kornaros acknowledges that his work was supported by the project "INVALOR: Research Infrastructure for Waste Valorization and Sustainable Management" (MIS 5002495) which is implemented under the Action "Reinforcement of the Research and Innovation Infrastructure", funded by the Operational Programme "Competitiveness, Entrepreneurship and Innovation” (NSRF 2014-2020) and co-financed by Greece and the European Union (European Regional Development Fund).

Special thanks to the talented Dr. Dimitrios Giannakoudakis who designed the graphical abstract.

\section{References}

[1] A. Ahmad, S.H. Mohd-Setapar, C.S. Chuong A. Khatoon, W.A. Wani, R. Kumar, M. Rafatullah, Recent advances in new generation dye removal technologies: nove search for approaches to reprocess wastewater, RSC Adv. 5 (2015) 30801-30818.

[2] B. Chen, M. Yuan, H. Liu, Removal of polycyclic aromatic hydrocarbons from aqueous solution using plant residue materials as a biosorbent, J. Hazard. Mater. 188 (2011) 436-442.

[3] V.K. Gupta, Suhas, application of low-cost adsorbents for dye removal-a review, J. Environ. Manag. 90 (2009) 2313-2342.

[4] R. Dutta, T.V. Nagarjuna, S.A. Mandavgane, J.D. Ekhe, Ultrafast removal of cationic dye using agrowaste-derived mesoporous adsorbent, Ind. Eng. Chem. Res. 53 (2014) 18558-18567.

[5] R.B. Arfi, S. Karoui, K. Mougin, A. Ghorbal, Adsorptive removal of cationic and anionic dyes from aqueous solution by utilizing almond shell as bioadsorbent, Euro-Mediterranean Journal for Environmental Integration 2 (2017) 20-32.

[6] A. Srinivasan, T. Viraraghavan, Decolorization of dye wastewaters by biosorbents: a review, J. Environ. Manag. 91 (2010) 1915-1929.

[7] S. Shoukat, H.N. Bhatti, M. Iqbal, S. Noreen, Mango stone biocomposite preparation and application for crystal violet adsorption: a mechanistic study, Microporous Mesoporous Mater. 239 (2017) 180-189.

[8] A.B. Albadarin, J. Mo, Y. Glocheux, S. Allen, G. Walker, C. Mangwandi, Preliminary investigation of mixed adsorbents for the removal of copper and methylene blue from aqueous solutions, Chem. Eng. J. 255 (2014) 525-534. 
[9] S. Antighin, L. Chirila, A. Popescu, Environmentally friendly techniques for wool dyeing process, Acta Chemica Iasi 23 (2015) 65-76.

[10] A.A. Atia, A.M. Donia, W.A. Al-Amrani, Adsorption/desorption behavior of acid orange 10 on magnetic silica modified with amine groups, Chem. Eng. J. 150 (2009) 55-62.

[11] F. Çolak, N. Atar, A. Olgun, Biosorption of acidic dyes from aqueous solution by Paenibacillus macerans: kinetic, thermodynamic and equilibrium studies, Chem. Eng. J. 150 (2009) 122-130.

[12] E. Ashtoukhy, Removal of color and cod from aqueous solution containing dyes by electrocoagulation in a new cell, Environ. Eng. Manag. J. (EEMJ) 13 (2014) 499-508.

[13] Y.-C. Lee, E.J. Kim, J.-W. Yang, H.-J. Shin, Removal of malachite green by adsorption and precipitation using aminopropyl functionalized magnesium phyllosilicate, J. Hazard. Mater. 192 (2011) 62-70.

[14] H. Ouni, M. Dhahbi, Spectrometric study of crystal violet in presence of polyacrylic acid and polyethylenimine and its removal by polyelectrolyte enhanced ultrafiltration, Sep. Purif. Technol. 72 (2010) 340-346.

[15] W.-E. Thung, S.-A. Ong, L.-N. Ho, Y.-S. Wong, F. Ridwan, H.K. Lehl, Y.-L. Oon, Y.-S. Oon, Biodegradation of Acid Orange 7 in a combined anaerobic-aerobic up-flow membrane-less microbial fuel cell: mechanism of biodegradation and electron transfer, Chem. Eng. J. 336 (2018) 397-405.

[16] Z. Yang, H. Yang, Z. Jiang, T. Cai, H. Li, H. Li, A. Li, R. Cheng, Flocculation of both anionic and cationic dyes in aqueous solutions by the amphoteric grafting flocculant carboxymethyl chitosan-graft-polyacrylamide, J. Hazard. Mater. 254 (2013) 36-45

[17] F.C. Moreira, R.A. Boaventura, E. Brillas, V.J. Vilar, Electrochemical advanced oxidation processes: a review on their application to synthetic and real wastewaters, Appl. Catal. B Environ. 202 (2017) 217-261.

[18] D. Clematis, G. Cerisola, M. Panizza, Electrochemical oxidation of a synthetic dye using a BDD anode with a solid polymer electrolyte, Electrochem. Commun. 75 (2017) 21-24.

[19] E. Sahinkaya, A. Sahin, A. Yurtsever, M. Kitis, Concentrate minimization and water recovery enhancement using pellet precipitator in a reverse osmosis process treating textile wastewater, J. Environ. Manag. 222 (2018) 420-427.

[20] M. Roushani, M. Mavaei, H.R. Rajabi, Graphene quantum dots as novel and green nano-materials for the visible-light-driven photocatalytic degradation of cationic dye, J. Mol. Catal. A Chem. 409 (2015) 102-109.

[21] A.H. Jawad, A.F. Alkarkhi, N.S.A. Mubarak, Photocatalytic decolorization of methylene blue by an immobilized TiO2 film under visible light irradiation: optimization using response surface methodology (RSM), Desalin. Water Treat. 56 (2015) 161-172.

[22] A.H. Jawad, N.S.A. Mubarak, M.A.M. Ishak, K. Ismail, W. Nawawi, Kinetics of photocatalytic decolourization of cationic dye using porous $\mathrm{TiO}_{2}$ film, Journal of Taibah University for Science 10 (2016) 352-362.

[23] H.R. Rajabi, H. Arjmand, S.J. Hoseini, H. Nasrabadi, Surface modified magnetic nanoparticles as efficient and green sorbents: synthesis, characterization, and application for the removal of anionic dye, J. Magn. Magn. Mater. 394 (2015) 7-13.

[24] I. Anastopoulos, I. Margiotoudis, I. Massas, The use of olive tree pruning waste compost to sequestrate methylene blue dye from aqueous solution, Int. J. Phytoremediation 20 (2018) 831-838.

[25] Q. Li, X. Tang, Y. Sun, Y. Wang, Y. Long, J. Jiang, H. Xu, Removal of rhodamine B from wastewater by modified Volvariella volvacea: batch and column study, RSC Adv. 5 (2015) 25337-25347

[26] S. Tahir, N. Rauf, Removal of a cationic dye from aqueous solutions by adsorption onto bentonite clay, Chemosphere 63 (2006) 1842-1848.

[27] B. Hameed, A. Ahmad, Batch adsorption of methylene blue from aqueous solution by garlic peel, an agricultural waste biomass, J. Hazard. Mater. 164 (2009) 870-875.

[28] I. Anastopoulos, G.Z. Kyzas, Agricultural peels for dye adsorption: a review of recent literature, J. Mol. Liq. 200 ( (2014) 381-389.

[29] P. Janoš, H. Buchtová, M. Rýznarová, Sorption of dyes from aqueous solutions onto fly ash, Water Res. 37 (2003) 4938-4944.

[30] S.G. Wang, Y. Ma, Y.J. Shi, W.X. Gong, Defluoridation performance and mechanism of nano-scale aluminum oxide hydroxide in aqueous solution, Journal of Chemical Technology \& Biotechnology: International Research in Process, Environmental \& Clean Technology 84 (2009) 1043-1050.

[31] A.E. Al Prol, M.A. El Azzem, A. Amer, M.E. El-Metwally, H.T.A. El-Hamid, K.M. ElMoselhy, Adsorption of cadmium (II) ions from aqueous solution onto mango leaves, Asian Journal of Physical and Chemical Sciences 2 (2017) 1-11.

[32] V.S. Tran, H.H. Ngo, W. Guo, J. Zhang, S. Liang, C. Ton-That, X. Zhang, Typical low cost biosorbents for adsorptive removal of specific organic pollutants from water, Bioresour. Technol. 182 (2015) 353-363.

[33] A. Abdolali, W. Guo, H.H. Ngo, S.-S. Chen, N.C. Nguyen, K.L. Tung, Typical lignocellulosic wastes and by-products for biosorption process in water and wastewater treatment: a critical review, Bioresour. Technol. 160 (2014) 57-66.

[34] I. Anastopoulos, A. Robalds, H.N. Tran, D. Mitrogiannis, D.A. Giannakoudakis, A. Hosseini-Bandegharaei, G.L. Dotto, Removal of heavy metals by leaves-derived biosorbents, Environ. Chem. Lett. (2018)https://doi.org/10.1007/s10311-01800829-X.

[35] D.A. Skoog, F.J. Holler, T.A. Nieman, Principios de Análisis Instrumental, fifth edition Mc Graw Hill Ed, Spain, 2013 592-596.

[36] D.A. Giannakoudakis, G.Z. Kyzas, A. Avranas, N.K. Lazaridis, Multi-parametric adsorption effects of the reactive dye removal with commercial activated carbons, J. Mol. Liq. 213 (2016) 381-389.

[37] M.S. Bretanha, M.C. Rochefort, G.L. Dotto, E.C. Lima, S.L. Dias, F.A. Pavan, Punica granatum husk (PGH), a powdered biowaste material for the adsorption of methylene blue dye from aqueous solution, Desalin. Water Treat. 57 (2016) 3194-3204.
[38] A. Khodabandehloo, A. Rahbar-Kelishami, H. Shayesteh, Methylene blue removal using Salix babylonica (weeping willow) leaves powder as a low-cost biosorbent in batch mode: kinetic, equilibrium, and thermodynamic studies, J. Mol. Liq. 244 (2017) 540-548

[39] S. Shrestha, Chemical, structural and elemental characterization of biosorbents using FE-SEM, SEMEDX, XRD/XRPD and ATR-FTIR Techniques, J. Chem. Eng. Process. Technol. 7 (2016) 1-11.

[40] P. Burdet, S.A. Croxall, P.A. Midgley, Enhanced quantification for 3D SEM-EDS: using the full set of available X-ray lines, Ultramicroscopy 148 (2015) 158-167.

[41] O.S. Bello, K.A. Adegoke, O.O. Akinyunni, Preparation and characterization of a novel adsorbent from Moringa oleifera leaf, Appl Water Sci 7 (2017) 1295-1305.

[42] K. Jyoti, A. Singh, Green synthesis of nanostructured silver particles and their catalytic application in dye degradation, J. Genet. Eng. Biotechnol. 14 (2016) 311-317.

[43] S. Baruah, A. Devi, K. Bhattacharyya, A. Sarma, Developing a biosorbent from Aegle marmelos leaves for removal of methylene blue from water, Int. J. Environ. Sci. Technol. 14 (2017) 341-352.

[44] D.A. Skoog, F.J. Holler, T.A. Nieman, Principios de Análisis Instrumental, fifth edition Mc Graw Hill Ed, Spain, 2013 409-412.

[45] H.S. Saroyan, D.A. Giannakoudakis, C.S. Sarafidis, N.K. Lazaridis, E.A. Deliyanni, Effective impregnation for the preparation of magnetic mesoporous carbon: application to dye adsorption, J. Chem. Technol. Biotechnol. 92 (2017) 1899-1911.

[46] D.A. Giannakoudakis, A. Hosseini-Bandegharaei, P. Tsafrakidou, K.S. Triantafyllidis, M. Kornaros, I. Anastopoulos, Aloe vera waste biomass-based adsorbents for the removal of aquatic pollutants: a review, J. Environ. Manag. 227 (2018) 354-364.

[47] L. Zhu, Y. Wang, T. He, L. You, X. Shen, Assessment of potential capability of water bamboo leaves on the adsorption removal efficiency of cationic dye from aqueous solutions, J. Polym. Environ. 24 (2016) 148-158.

[48] D.A. Giannakoudakis, M. Seredych, E. Rodríguez-Castellón, T.J. Bandosz, Mesoporous graphitic carbon nitride-based nanospheres as visible-light active chemical warfare agents decontaminant, ChemNanoMat 2 (2016) 268-272.

[49] L. Lim, N. Priyantha, N.M. Zaidi, A superb modified new adsorbent, Artocarpus odoratissimus leaves, for removal of cationic methyl violet 2B dye, Environ. Earth Sci. 75 (2016) 1179.

[50] A.K. Mishra, Nanomaterials for Water Remediation: Carbon-based Materials, Vol. 1, Smithers Rapra Technology Ltd., United Kingdom, 2016 50-51.

[51] A.C. Silva, A.O. Jorgetto, M.H. Wondracek, M.J. Saeki, J.F. Schneider, V.A. Pedrosa, M.A. Martines, G.R. Castro, Characterization of corn (Zea mays) leaf powder and its adsorption properties regarding $\mathrm{Cu}$ (II) and Cd (II) from aqueous samples, Bioresources 10 (2014) 1099-1114.

[52] I. Michalak, K. Chojnacka, A. Witek-Krowiak, State of the art for the biosorption process-a review, Appl. Biochem. Biotechnol. 170 (2013) 1389-1416.

[53] J. Jagiello, Stable numerical solution of the adsorption integral equation using splines, Langmuir 10 (1994) 2778-2785.

[54] C.O. Ania, T.J. Bandosz, Importance of structural and chemical heterogeneity of activated carbon surfaces for adsorption of dibenzothiophene, Langmuir 21 (2005) 7752-7759.

[55] A.A. Spagnoli, D.A. Giannakoudakis, S. Bashkova, Adsorption of methylene blue on cashew nut shell based carbons activated with zinc chloride: the role of surface and structural parameters, J. Mol. Liq. 229 (2017) 465-471.

[56] R. Rehman, T. Mahmud, M. Irum, Brilliant green dye elimination from water using Psidium guajava leaves and Solanum tuberosum peels as adsorbents in environmentally benign way, J. Chem. 2015 (2015) 126036.

[57] S.R. Byrn, G. Zografi, X.S. Chen, Differential scanning calorimetry and thermogravimetric analysis, Solid-State Properties of Pharmaceutical Materials, John Wiley \& Sons, USA, 2017.

[58] K. Hemashree, J.I. Bhat, Synthesis, characterization and adsorption behavior of coconut leaf carbon, Res. Chem. Intermed. 43 (2017) 4369-4386.

[59] N. Ben Douissa, S. Dridi-Dhaouadi, M.F. Mhenni, Spectrophotometric investigation of the interactions between cationic (CI Basic Blue 9) and anionic ( $\mathrm{Cl}$ Acid Blue 25) dyes in adsorption onto extracted cellulose from Posidonia oceanic in single and binary system, Water Sci. Technol. 73 (2016) 2211-2221.

[60] S.N. Jain, P.R. Gogate, Acid Blue 113 removal from aqueous solution using novel biosorbent based on $\mathrm{NaOH}$ treated and surfactant modified fallen leaves of Prunus Dulcis, J. Environ. Chem. Eng. 5 (2017) 3384-3394.

[61] Y. Yan, B. Xiang, X. Yi, Y. Li, Q. Jia, Competitive adsorption of acid dyes from aqueous solution on diethylenetriamine-modified chitosan beads, J. Appl. Polym. Sci. 131 (2014).

[62] M.A. Behnajady, H. Eskandarloo, F. Eskandarloo, Artificial neural network modeling of the influence of sol-gel synthesis variables on the photocatalytic activity of $\mathrm{TiO}_{2}$ nanoparticles in the removal of Acid Red 27, Res. Chem. Intermed. 41 (2015) 6463-6476.

[63] P. Vijayalakshmi, V.S.S. Bala, K. Thiruvengadaravi, P. Panneerselvam, M. Palanichamy, S. Sivanesan, Removal of acid violet 17 from aqueous solutions by adsorption onto activated carbon prepared from pistachio nut shell, Sep. Sci. Technol. 46 (2010) 155-163.

[64] O. Moradi, Adsorption behavior of basic red 46 by single-walled carbon nanotubes surfaces, Fullerenes, Nanotubes, Carbon Nanostruct. 21 (2013) 286-301.

[65] J. Tang, B. Mu, W. Wang, M. Zheng, A. Wang, Fabrication of manganese dioxide/carbon/attapulgite composites derived from spent bleaching earth for adsorption of $\mathrm{Pb}$ (ii) and brilliant green, RSC Adv. 6 (2016) 36534-36543.

[66] H. Zhang, H. Chen, S. Azat, Z.A. Mansurov, X. Liu, J. Wang, X. Su, R. Wu, Super adsorption capability of rhombic dodecahedral Ca-Al layered double oxides for Congo red removal, J. Alloys Compd. 768 (2018) 572-581.

[67] P. Lu, X. Hu, Y. Li, M. Zhang, X. Liu, Y. He, F. Dong, M. Fu, Z. Zhang, One-step preparation of a novel $\mathrm{SrCO}_{3} / \mathrm{gC}_{3} \mathrm{~N}_{4}$ nano-composite and its application in selective adsorption of crystal violet, RSC Adv. 8 (2018) 6315-6325. 
[68] I. Khurana, A.K. Shaw, J.M. Khurana, P.K. Rai, Batch and dynamic adsorption of Eriochrome Black T from water on magnetic graphene oxide: experimental and theoretical studies, J. Environ. Chem. Eng. 6 (2018) 468-477.

[69] L.B. Escudero, E. Agostini, G.L. Dotto, Application of tobacco hairy roots for the removal of malachite green from aqueous solutions: experimental design, kinetic, equilibrium, and thermodynamic studies, Chem. Eng. Commun. 205 (2018) $122-133$.

[70] G. Dotto, F. Rodrigues, E. Tanabe, R. Fröhlich, D. Bertuol, T. Martins, E. Foletto, Development of chitosan/bentonite hybrid composite to remove hazardous anionic and cationic dyes from colored effluents, J. Environ. Chem. Eng. 4 (2016) 3230-3239.

[71] D.L. Postai, C.A. Demarchi, F. Zanatta, D.C.C. Melo, C.A. Rodrigues, Adsorption of rhodamine B and methylene blue dyes using waste of seeds of Aleurites moluccana, a low cost adsorbent, Alex. Eng. J. 55 (2016) 1713-1723.

[72] R. Kosheleva, A.C. Mitropoulos, G.Z. Kyzas, Activated carbon from food waste, in: G Crini, E. Lichtfouse (Eds.), Green Adsorbents for Pollutant Removal: Innovative Materials, Springer International Publishing, Cham 2018, pp. 159-182.

[73] C.N. Delgado, R. Mendez, Preparation of carbon materials from lignocellulosic biomass, in: T.E. Rufford, D. Hulicova-Jurcakova, J. Zhu (Eds.), Green Carbon Materials: Advances and Applications, Taylor and Francis Group, US 2011, p. 40.

[74] K. Foo, B.H. Hameed, An overview of dye removal via activated carbon adsorption process, Desalin. Water Treat. 19 (2010) 255-274.

[75] D. Das, D.P. Samal, B. Meikap, Preparation of activated carbon from green coconut shell and its characterization, Journal of Chemical Engineering \& Process Technology 6 (2015) 1.

[76] V. Gupta, P. Carrott, R. Singh, M. Chaudhary, S. Kushwaha, Cellulose: a review as natural, modified and activated carbon adsorbent, Bioresour. Technol. 216 (2016) 1066-1076.

[77] N.K. Tripathi, V.V. Singh, M. Sathe, V.B. Thakare, B. Singh, Activated carbon fabric: an adsorbent material for chemical protective clothing, Def. Sci. J. 68 (2018) 83-90.

[78] Z. Jeirani, C.H. Niu, J. Soltan, Adsorption of emerging pollutants on activated carbon, Rev. Chem. Eng. 33 (2017) 491-522.

[79] M.T. Yagub, T.K. Sen, S. Afroze, H.M. Ang, Dye and its removal from aqueous solution by adsorption: a review, Adv. Colloid Interf. Sci. 209 (2014) 172-184.

[80] G. Kyzas, J. Fu, K. Matis, The change from past to future for adsorbent materials in treatment of dyeing wastewaters, Materials 6 (2013) 5131-5158.

[81] E.A. Deliyanni, G.Z. Kyzas, K.S. Triantafyllidis, K.A. Matis, Activated carbons for the removal of heavy metal ions: a systematic review of recent literature focused on lead and arsenic ions, Open Chem. 13 (2015).

[82] I. Anastopoulos, A. Robalds, H.N. Tran, D. Mitrogiannis, D.A. Giannakoudakis, A Hosseini-Bandegharaei, G.L. Dotto, Leaf Biosorbents for the Removal of Heavy Metals, Green Adsorbents for Pollutant Removal, Springer, 2018 87-126.

[83] W.W. Ngah, M. Hanafiah, Removal of heavy metal ions from wastewater by chemically modified plant wastes as adsorbents: a review, Bioresour. Technol. 99 (2008) 3935-3948.

[84] G.Z. Kyzas, M. Kostoglou, Green adsorbents for wastewaters: a critical review, Dent. Mater. 7 (2014) 333-364.

[85] H.N. Tran, S.-J. You, A. Hosseini-Bandegharaei, H.-P. Chao, Mistakes and inconsistencies regarding adsorption of contaminants from aqueous solutions: a critical review, Water Res. 120 (2017) 88-116 (doi: 110.1016/j.watres.2017.1004.1014).

[86] I. Guerrero-Coronilla, L. Morales-Barrera, T.L. Villegas-Garrido, E. Cristiani-Urbina, Biosorption of amaranth dye from aqueous solution by roots, leaves, stems and the whole plant of E. crassipes, Environ. Eng. Manag. J. 14 (2014) 1917-1926.

[87] N. Gupta, A.K. Kushwaha, M.C. Chattopadhyaya, Adsorption studies of cationic dyes onto Ashoka (Saraca asoca) leaf powder, J. Taiwan Inst. Chem. Eng. 43 (2012) 604-613.

[88] S.N. Jain, P.R. Gogate, Adsorptive removal of acid violet 17 dye from wastewater using biosorbent obtained from $\mathrm{NaOH}$ and $\mathrm{H}_{2} \mathrm{SO}_{4}$ activation of fallen leaves of Ficus racemosa, J. Mol. Liq. 243 (2017) 132-143.

[89] L. Cavas, Z. Karabay, H. Alyuruk, H. Doğan, G.K. Demir, Thomas and artificial neural network models for the fixed-bed adsorption of methylene blue by a beach waste Posidonia oceanica (L.) dead leaves, Chem. Eng. J. 171 (2011) 557-562.

[90] K.G. Bhattacharyya, A. Sharma, Kinetics and thermodynamics of methylene blue adsorption on neem (Azadirachta indica) leaf powder, Dyes Pigments 65 (2005) 51-59.

[91] G.K. Demir, M.U. Dural, H. Alyuruk, L. Cavas, Artificial neural network model for biosorption of methylene blue by dead leaves of Posidonia oceanica (L.) Delile, Neural Network World 22 (2012) 479.

[92] R. Han, W. Zou, W. Yu, S. Cheng, Y. Wang, J. Shi, Biosorption of methylene blue from aqueous solution by fallen phoenix tree's leaves, J. Hazard. Mater. 141 (2007) 156-162.

[93] K. Polipalli, K. Pulipati, Equilibrium, kinetic and thermodynamic studies of biosorption of methylene blue dye using plant biomass as biosorbent: optimization using response surface methodology (RSM), International Journal of Scientific \& Engineering Research 4 (2013) 1244-1252.

[94] V. Ponnusami, V. Gunasekar, S. Srivastava, Kinetics of methylene blue removal from aqueous solution using gulmohar (Delonix regia) plant leaf powder: multivariate regression analysis, J. Hazard. Mater. 169 (2009) 119-127.

[95] C.-H. Weng, Y.-T. Lin, T.-W. Tzeng, Removal of methylene blue from aqueous solution by adsorption onto pineapple leaf powder, J. Hazard. Mater. 170 (2009) 417-424.

[96] A.H. Jawad, R.A. Rashid, R.M. Mahmuod, M.A.M. Ishak, N.N. Kasim, K. Ismail, Adsorption of methylene blue onto coconut (Cocos nucifera) leaf: optimization, isotherm and kinetic studies, Desalin. Water Treat. 57 (2016) 8839-8853.

[97] S. Cengiz, L. Cavas, A promising evaluation method for dead leaves of Posidonia oceanica (L.) in the adsorption of methyl violet, Mar. Biotechnol. 12 (2010) 728-736.
[98] P.D. Saha, S. Chakraborty, S. Chowdhury, Batch and continuous (fixed-bed column) biosorption of crystal violet by Artocarpus heterophyllus (jackfruit) leaf powder, Colloids Surf. B: Biointerfaces 92 (2012) 262-270.

[99] F. Deniz, S.D. Saygideger, Equilibrium, kinetic and thermodynamic studies of acid Orange 52 dye biosorption by Paulownia tomentosa Steud. leaf powder as a lowcost natural biosorbent, Bioresour. Technol. 101 (2010) 5137-5143.

[100] I. Guerrero-Coronilla, L. Morales-Barrera, E. Cristiani-Urbina, Kinetic, isotherm and thermodynamic studies of amaranth dye biosorption from aqueous solution onto water hyacinth leaves, J. Environ. Manag. 152 (2015) 99-108.

[101] F. Deniz, S. Karaman, Removal of Basic Red 46 dye from aqueous solution by pine tree leaves, Chem. Eng. J. 170 (2011) 67-74.

[102] F. Deniz, S.D. Saygideger, Removal of a hazardous azo dye (Basic Red 46) from aqueous solution by princess tree leaf, Desalination 268 (2011) 6-11.

[103] K.G. Bhattacharyya, A. Sarma, Adsorption characteristics of the dye, brilliant green, on neem leaf powder, Dyes Pigments 57 (2003) 211-222.

[104] P. Kalpana, V. Omkar, P. King, Removal of congo red fromaqueous solutions using plant biomass: equilibrium and kinetic studies, Chem. Technol. 11 (2016) 43-50.

[105] V. Karthik, M. Velan, M.D. Kumar, S. Renganathan, Removal of Brill Red 5B from an aqueous solution using Cicca acida biomass, Can. J. Chem. Eng. 87 (2009) 554-561.

[106] M. Peydayesh, A. Rahbar-Kelishami, Adsorption of methylene blue onto Platanus orientalis leaf powder: kinetic, equilibrium and thermodynamic studies, J. Ind Eng. Chem. 21 (2015) 1014-1019.

[107] R. Han, L. Zhang, C. Song, M. Zhang, H. Zhu, L. Zhang, Characterization of modified wheat straw, kinetic and equilibrium study about copper ion and methylene blue adsorption in batch mode, Carbohydr. Polym. 79 (2010) 1140-1149.

[108] S.M.G. Saeed, S.A. Sayeed, S. Ashraf, A. Qureshi, R. Ali, R. Kausar, Z.S. Saify Amaranth-protein interaction in food system and its impact on tryptic digestibility, J. Chem. Soc. Pak. 34 (2013).

[109] O.S. Bello, S. Banjo, Equilibrium, kinetic, and quantum chemical studies on the adsorption of Congo red using Imperata cylindrica leaf powder activated carbon, Toxicol. Environ. Chem. 94 (2012) 1114-1124.

[110] V. Gunasekar, V. Ponnusami, Kinetics, equilibrium, and thermodynamic studies on adsorption of methylene blue by carbonized plant leaf powder, J. Chem. 2012 (2013).

[111] A.H. Jawad, R.A. Rashid, M.A.M. Ishak, L.D. Wilson, Adsorption of methylene blue onto activated carbon developed from biomass waste by $\mathrm{H}_{2} \mathrm{SO}_{4}$ activation: kinetic, equilibrium and thermodynamic studies, Desalin. Water Treat. 57 (2016) 25194-25206.

[112] R.A. Rashid, A. Jawad, M. Azlan, M. Ishak, N.N. Kasim, $\mathrm{FeCl}_{3}$-activated carbon developed from coconut leaves: characterization and application for methylene blue removal, Sains Malaysiana 47 (2018) 603-610.

[113] S. Ghosh, A. Bandyopadhyay, Adsorption of methylene blue onto citric acid treated carbonized bamboo leaves powder: equilibrium, kinetics, thermodynamics analyses, J. Mol. Liq. 248 (2017) 413-424.

[114] A.H. Jawad, S. Sabar, M.A.M. Ishak, L.D. Wilson, S.S. Ahmad Norrahma, M. Talari, A.M. Farhan, Microwave-assisted preparation of mesoporous-activated carbon from coconut (Cocos nucifera) leaf by $\mathrm{H}_{3} \mathrm{PO}_{4}$ activation for methylene blue adsorption, Chem. Eng. Commun. 204 (2017) 1143-1156.

[115] R.A. Rashid, A.H. Jawad, M.A.M. Ishak, N.N. Kasim, KOH-activated carbon developed from biomass waste: adsorption equilibrium, kinetic and thermodynamic studies for methylene blue uptake, Desalin. Water Treat. 57 (2016) 27226-27236.

[116] A.T. Ojedokun, O.S. Bello, Kinetic modeling of liquid-phase adsorption of Congo red dye using guava leaf-based activated carbon, Appl Water Sci 7 (2017) 1965-1977.

[117] A. Khan, X. Wang, K. Gul, F. Khuda, Z. Aly, A. Elseman, Microwave-assisted spent black tea leaves as cost-effective and powerful green adsorbent for the efficient removal of Eriochrome black T from aqueous solutions, Egyptian J. Basic Appl. Sci. 5 (2018) 171-182.

[118] M. Ahmaruzzaman, M.J.K. Ahmed, S. Begum, Remediation of Eriochrome black Tcontaminated aqueous solutions utilizing H3PO4-modified berry leaves as a nonconventional adsorbent, Desalin. Water Treat. 56 (2015) 1507-1519.

[119] Z.M. Hussin, N. Talib, N.M. Hussin, M.A. Hanafiah, W.K. Khalir, Methylene blue adsorption onto $\mathrm{NaOH}$ modified durian leaf powder: isotherm and kinetic studies, American J. Environ. Eng. 5 (2015) 38-43.

[120] L. Gong, W. Sun, L. Kong, Adsorption of methylene blue by NaOH-modified dead leaves of plane trees, Computational Water, Energy, and Environmental Engineering 2 (2013) 13-19.

[121] S. Jain, P. Gogate, NaOH-treated dead leaves of Ficus racemosa as an efficient biosorbent for Acid Blue 25 removal, Int. J. Environ. Sci. Technol. 14 (2017) 531-542.

[122] M. Makeswari, T. Santhi, M. Ezhilarasi, Adsorption of methylene blue dye by citric acid modified leaves of Ricinus communis from aqueous solutions, J. Chem. Pharm. Res. 8 (2016) 452-462.

[123] M. Goswami, P. Phukan, Enhanced adsorption of cationic dyes using sulfonic acid modified activated carbon, J. Environ. Chem. Eng. 5 (2017) 3508-3517.

[124] A.A. Kamaru, N.S. Sani, N.A.N.N. Malek, Raw and surfactant-modified pineapple leaf as adsorbent for removal of methylene blue and methyl orange from aqueous solution, Desalin. Water Treat. 57 (2016) 18836-18850.

[125] E.O. Oyelude, U.R. Owusu, Adsorption of methylene blue from aqueous solution using acid modified Calotropis procera leaf powder, J. Appl. Sci. Environ. Sanit 6 (2011) 477-484.

[126] Y. Tanga, Y. Lia, Y. Zhaoa, Q. Zhoua, Y. Pengb, Enhanced removal of methyl violet using $\mathrm{NaOH}$-modified $\mathrm{C}$. camphora leaves powder and its renewable adsorption, Desalin. Water Treat. 98 (2017) 306-314.

[127] J.-X. Yang, G.-B. Hong, Adsorption behavior of modified Glossogyne tenuifolia leaves as a potential biosorbent for the removal of dyes, J. Mol. Liq. 252 (2018) 289-295. 
[128] P.S. Ghosal, A.K. Gupta, Determination of thermodynamic parameters from Langmuir isotherm constant-revisited, J. Mol. Liq. 225 (2017) 137-146.

[129] M.A. Al-Anber, Thermodynamics approach in the adsorption of heavy metals, in: J.C. Moreno-Pirajan (Ed.), Thermodynamics - Interaction Studies - Solids, Liquids and Gases, InTech, Rijeka, Croatia 2011, pp. 737-764.

[130] D. Park, Y.-S. Yun, J.M. Park, The past, present, and future trends of biosorption, Biotechnol. Bioprocess Eng. 15 (2010) 86-102

[131] K.M. Doke, E.M. Khan, Adsorption thermodynamics to clean up wastewater; critical review, Rev. Environ. Sci. Biotechnol. 12 (2013) 25-44.

[132] B. Fu, C. Ge, L. Yue, J. Luo, D. Feng, H. Deng, H. Yu, Characterization of biochar derived from pineapple peel waste and its application for sorption of oxytetracycline from aqueous solution, Bioresources 11 (2016) 9017-9035

[133] T. Nharingo, M. Moyo, Application of Opuntia ficus-indica in bioremediation of wastewaters. A critical review, J. Environ. Manag. 166 (2016) 55-72.

[134] I. Anastopoulos, G.Z. Kyzas, Are the thermodynamic parameters correctly estimated in liquid-phase adsorption phenomena? J. Mol. Liq. 218 (2016) 174-185 (doi: 110.1016/j.molliq.2016.1002.1059).

[135] I. Anastopoulos, A. Bhatnagar, B.H. Hameed, Y.S. Ok, M. Omirou, A review on wastederived adsorbents from sugar industry for pollutant removal in water and wastewater, J. Mol. Liq. 240 (2017) 179-188.
[136] I. Anastopoulos, A. Bhatnagar, E.C. Lima, Adsorption of rare earth metals: a review of recent literature, J. Mol. Liq. 221 (2016) 954-962.

[137] Y. Chang, J.-Y. Lai, D.-J. Lee, Thermodynamic parameters for adsorption equilibrium of heavy metals and dyes from wastewaters: research updated, Bioresour. Technol. 222 (2016) 513-516.

[138] A. Ramesh, D. Lee, J. Wong, Thermodynamic parameters for adsorption equilibrium of heavy metals and dyes from wastewater with low-cost adsorbents, J. Colloid Interface Sci. 291 (2005) 588-592.

[139] X. Liu, D.-J. Lee, Thermodynamic parameters for adsorption equilibrium of heavy metals and dyes from wastewaters, Bioresour. Technol. 160 (2014) 24-31.

[140] S.N. Jain, P.R. Gogate, Efficient removal of Acid Green 25 dye from wastewater using activated Prunus dulcis as biosorbent: batch and column studies, J. Environ. Manag. 210 (2018) 226-238.

[141] V. Gunasekar, V. Ponnusami, Kinetics, equilibrium, and thermodynamic studies on adsorption of methylene blue by carbonized plant leaf powder, J. Chem. 2012 (2013) 1-6.

[142] X. Han, W. Wang, X. Ma, Adsorption characteristics of methylene blue onto low cost biomass material lotus leaf, Chem. Eng. J. 171 (2011) 1-8. 